\title{
Recherches sur l'écologie des Hydracariens (Hydrachnellae, Acari) dans les eaux courantes
}

\author{
E. Angelier ${ }^{1}$ \\ M.-L. Angelier ${ }^{1}$ \\ J. Lauga ${ }^{2}$
}

Mots clés : Limnologie, Eaux courantes, Ecologie, Biogéographie, théorie des îles, Hydrachnellae.

Le peuplement en Hydracariens (Hydrachnellae, Acari) a été étudié dans 108 stations (86 espèces) des Pyrénées centrales. L'analyse canonique et l'analyse factorielle des correspondances permettent de mettre en évidence :

- d'une part, une corrélation entre les facteurs écologiques habituellement reconnus (température, vitesse du courant,...) et un certain nombre de parametres topographiques, hydrologiques, géologiques,..., qui constituent un complexe stationnel, stable dans le temps pour une mème station.

- d'autre part, une corrélation entre les complexes stationnels et un certain nombre (8) d'associations d'Hydracariens. Cinq paramètres, - altitude de la source, altitude de la station, pente, surface du bassin-versant et régime des eaux, suffisent à définir un complexe stationnel et à expliquer, avec la nature du substrat (galets ou mousses), la distribution des espèces. Le régime des eaux, notamment, joue un rôle fondamental dans cette distribution et justifie la distinction des auteurs de langue allemande entre faune de Mittel - et Hochgebirge.

En fonction du substrat, du taux de reproduction, du cycle biologique, différents types biologiques d'Hydracariens sont mis en évidence. Mais, pour expliquer la structure des communautés, et notamment leur diversité, il faut faire appel à des notions comme la stabilité du biotope (durée), sa surface (espace). la distance entre biotopes équivalents, - c'est-àdire à la théorie des îles de Mac-Arthur et Wilson. Le peuplement des biotopes instables, de surface limitée et isolés est à la limite aléatoire.

Resarch on the ecology of water-mites (Hydrachnellae, Acari) in flowing water.

Keywords : Limnology, Flowing water, Ecology, Biogeography, Theory of islands, Hydrachnellae.

Populations of water-mites (Hydrachnellae, Acari) have been studied at 108 stations ( 86 species) in the central Pyrenees.

Canonical analysis and factorial analysis have revealed :

- on the one hand, a correlation between well known ecological factors (temperature, current speed, etc) and a certain number of topographical, hydrological, geological parameters which form a locality complex, that is stable in time at each station:

- on the other hand, a correlation between the locality complexes and a certain number (8) of water-mite associations. Five parameters (altitude of the source, altitude of the station, slope, catchment area, and water regime) are sufficient to define the locality complex and to explain, together with the type of substratum (gravel or moss), the species distribution. The water regime, notably, plays a fundamental role in this distribution and justifies the distinction of German-speaking authors between the fauna of Mittel-and Hochgebirge.

Different biological types of water-mites are identified as a function of substratum, rate of reproduction and biological cycle. But, to explain the structure of communities and especially their diversity, it is necessary to take account of other factors such as biotope stability (time), its area (space), the distance between equivalent biotopes, - that is to say MacArthur and Wilson's theory of islands. Populations of unstable biotopes, of limited areas and isolated are at the uncertain limit.

\section{Sommaire}

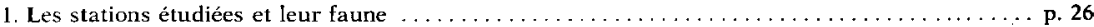

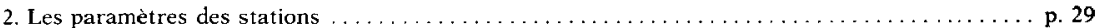

3. Les associations d'Hydracariens et les relations espèces-paramètres des milieux . . . . . . . p. 37

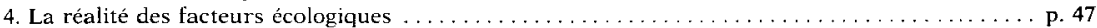

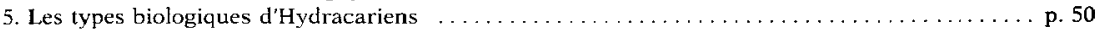

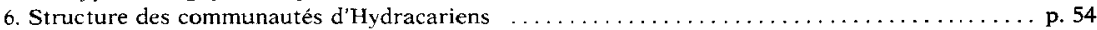

7. Equilibre dynamique des peuplements d'eaux courantes $\ldots \ldots \ldots \ldots \ldots \ldots \ldots \ldots \ldots \ldots \ldots \ldots \ldots \ldots \ldots$

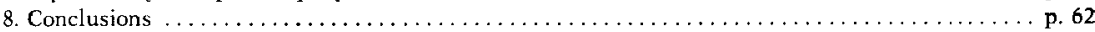

1. Laboratoire d'Hydrobiologie, U.A. 695, C.N.R.S., Université Paul Sabatier, 118, route de Narbonne, 31062 Toulouse Cédex (France)

2. Centre d'Étude des Ressources Renouvelables C.N.R.S., 29, rue Jeanne Marvig. 31055 Toulouse Cédex (France). 


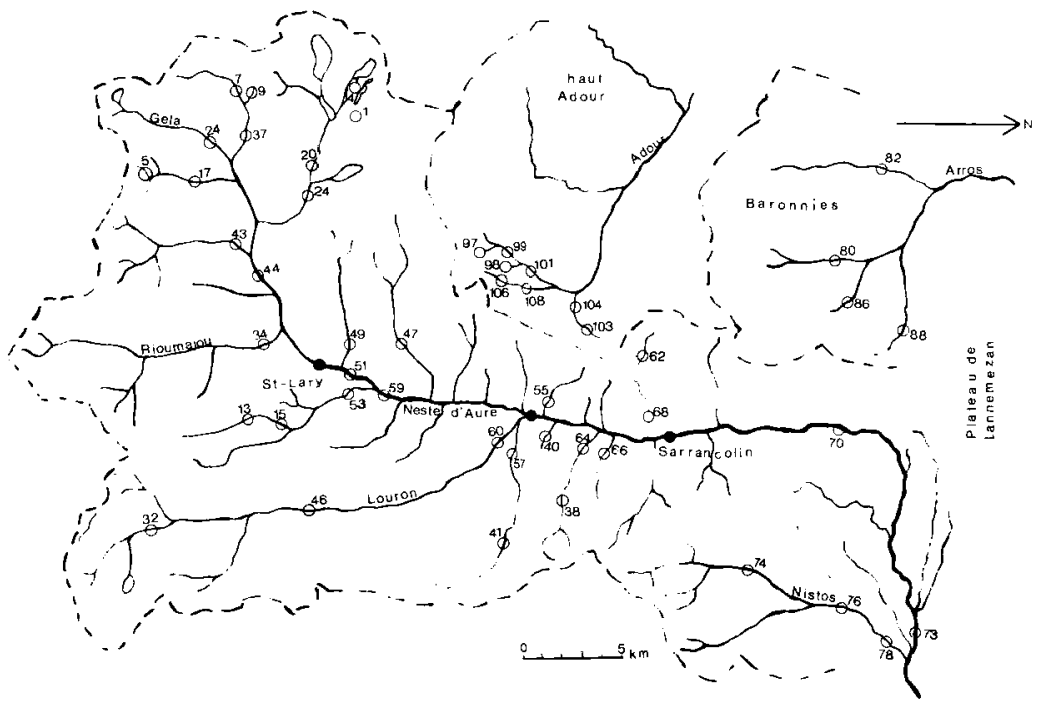

Fig. 1. Stations prospectées dans la vallée d'Aure, le haut Adour et les Baronnies. Les rivières des Coteaux de Gascogne prennent leur source sur le plateau de Lannemezan.

Des recherches menées depuis 1975 avaient pour premier objet de compléter nos connaissances sur les Hydracariens des eaux courantes des Pyrénées centrales. Elles avaient également pour but :

- de déterminer les relations entre les paramètres physico-chimiques habituellement mesurés de façon instantanée (température, vitesse du courant, $\mathrm{pH}, .$. ) et des paramètres topographiques, hydrologiques, géologiques,... Ces derniers constituent un complexe stationnel, stable dans le temps, indépendant d'évènements fortuits ou saisonniers.

- de mettre en évidence des groupements et leur corrélation avec les complexes stationnels.

- de comprendre les mécanismes de la colonisation des eaux courantes par les Hydracariens.
Ce travail est fondé sur 108 stations des Pyrénées centrales et Coteaux de Gascogne, et 86 espèces.

\section{1. - Les stations étudiées et leur faune}

\subsection{Les stations étudiées (fig. 1, tableau I)}

Une station correspond à une surface dont les paramètres écologiques sont homogènes. Decamps (1967) et Thomas (1976) ont donné les caractéristiques des eaux courantes de la vallée d'Aure. Nous ne donnerons donc ici que celles de la haute vallée de l'Adour, des Baronnies et des Coteaux de Gascogne. Les dates indiquées pour chaque station (tableau I) correspondent aux prélèvements de fin d'été et début d'automne, seuls utilisés dans les analyses statistiques. 
Tableau I. Les stations prospectées.

\begin{tabular}{|c|c|c|c|c|}
\hline COURS D'EAU & ALTITUDE & COURANT & SUBSTRAT & DATE \\
\hline 1 Tourbière Despax & $2200 \mathrm{~m}$ & rapide & mousses & 19.9 .76 \\
\hline ". & ", & $\begin{array}{l}\text { lent } \\
\text { très lent }\end{array}$ & $"$ & $"$ \\
\hline 4 Aumar (ruisselet) & $2200 \mathrm{~m}$ & vif & mousses & 19.9 .76 \\
\hline $\begin{array}{l}5 \text { Neste de Saux (r. Hourquet) } \\
6\end{array}$ & $1800 \mathrm{~m}$ & $\begin{array}{l}\text { rapide } \\
\text { rapide }\end{array}$ & $\begin{array}{l}\text { pierres } \\
\text { mousses }\end{array}$ & 1.9 .76 \\
\hline 7 Neste de Badet & $1800 \mathrm{~m}$ & rapide & mousses & 28.8 .76 \\
\hline 8 Source (affluent Badet) & $1800 \mathrm{~m}$ & violent & mousses & 28.8 .76 \\
\hline 9 ruisselet (affluent Badet) & $1800 \mathrm{~m}$ & viol. (casc) & mousses & 28.8 .76 \\
\hline 10 & & rapide & & " \\
\hline 11 & " & lent & cailloux & $"$ \\
\hline 12 vasque (affluent Badet) & $1800 \mathrm{~m}$ & lent & mousses & 28.8 .76 \\
\hline $\begin{array}{l}13 \text { r. d'Arsoue (La Mousquère) } \\
14\end{array}$ & $1220 \mathrm{~m}$ & $\underset{"}{\text { violent }}$ & $\begin{array}{l}\text { mous. hygr. } \\
\text { mousses }\end{array}$ & 29.8 .76 \\
\hline $15 \quad "$ & $1100 \mathrm{~m}$ & vif & cailloux & $"$ \\
\hline 16 & " & vif & mousses & $"$ \\
\hline $17 \quad " \prime$ & " & lent & mousses & $"$ \\
\hline $\begin{array}{l}18 \text { Neste de Saux } \\
19\end{array}$ & $1550 \mathrm{~m}$ & $\begin{array}{l}\text { vif } \\
\text { vif }\end{array}$ & $\begin{array}{l}\text { cailloux } \\
\text { mousses }\end{array}$ & 1.9 .76 \\
\hline $\begin{array}{l}20 \text { Source à Artigusse } \\
21\end{array}$ & $1559 \mathrm{~m}$ & $\begin{array}{l}\text { vif } \\
\text { vif }\end{array}$ & mousses & $\begin{array}{l}19.9 .76 \\
. "\end{array}$ \\
\hline 22 Neste de Couplan (Artigusse) & $1559 \mathrm{~m}$ & $\begin{array}{l}\text { vif } \\
\text { vif }\end{array}$ & $\begin{array}{l}\text { cailloux } \\
\text { mous. (casc) }\end{array}$ & $"$ \\
\hline $\begin{array}{l}24 \text { Neste de la Gela } \\
25\end{array}$ & $1400 \mathrm{~m}$ & $\begin{array}{l}\text { vif } \\
\text { vif }\end{array}$ & $\begin{array}{l}\text { cailloux } \\
\text { mous. (case) }\end{array}$ & $\begin{array}{c}2.9 .76 \\
. "\end{array}$ \\
\hline $26 \quad 11$ & $"$ & vif & mousses & " \\
\hline $\begin{array}{l}27 \text { Neste de Couplan } \\
28\end{array}$ & $1380 \mathrm{~m}$ & $\begin{array}{l}\text { rapide } \\
\text { rapide }\end{array}$ & $\begin{array}{l}\text { pierres } \\
\text { mousses }\end{array}$ & $\begin{array}{c}6.10 .76 \\
" 1\end{array}$ \\
\hline $\begin{array}{l}29 \text { Source de Couplan (affluent) } \\
30\end{array}$ & $1380 \mathrm{~m}$ & $\begin{array}{l}\text { lent } \\
\text { lent }\end{array}$ & $\begin{array}{l}\text { cailloux } \\
\text { mousses }\end{array}$ & 23.12 .77 \\
\hline $\begin{array}{l}31 \text { Neste de Couplan } \\
32 \text { Neste du Louron } \\
33\end{array}$ & $\begin{array}{l}1380 \mathrm{~m} \\
1230 \mathrm{~m} \\
" \prime\end{array}$ & $\begin{array}{l}\text { rapide } \\
\text { rapide }\end{array}$ & $\begin{array}{l}\text { mousses } \\
\text { pierres } \\
\text { mousses }\end{array}$ & $\begin{array}{l}23.12 .77 \\
16.10 .76\end{array}$ \\
\hline $\begin{array}{l}34 \text { Neste de Rioumajou } \\
35\end{array}$ & $1130 \mathrm{~m}$ & $\underset{n}{\text { violent }}$ & $\begin{array}{l}\text { pierres } \\
\text { mousses }\end{array}$ & 6.10 .76 \\
\hline $36 \quad " 1$ & $"$ & rapide & mous. hygr. & " \\
\hline $\begin{array}{l}37 \text { Neste de Badet } \\
38 \mathrm{r} \text {. d'Ardengost } \\
39\end{array}$ & $\begin{array}{l}1400 \mathrm{~m} \\
1075 \mathrm{~m} \\
\end{array}$ & vif & $\begin{array}{l}\text { pierres } \\
\text { pierres } \\
\text { mousses }\end{array}$ & $\begin{array}{l}2.9 .76 \\
30.8 .76 \\
\prime \prime\end{array}$ \\
\hline $\begin{array}{l}40 \text { r. de Garrade } \\
41 \text { Le Lastie . } \\
42\end{array}$ & $\begin{array}{c}875 \mathrm{~m} \\
1066 \mathrm{~m} \\
\text { " }\end{array}$ & $\begin{array}{l}\text { rapide } \\
\text { rapide }\end{array}$ & $\begin{array}{l}\text { mousses } \\
\text { pierres } \\
\text { mousses }\end{array}$ & $\begin{array}{l}30.8 .76 \\
6.9 .75 \\
"\end{array}$ \\
\hline $\begin{array}{l}43 \text { Neste de Moudang } \\
44 \text { Neste d'Aure à Eget } \\
45\end{array}$ & $\begin{array}{l}1053 \mathrm{~m} \\
1020 \mathrm{~m} \\
=\end{array}$ & $\begin{array}{l}\text { rapide } \\
\text { vif } \\
\text { vif }\end{array}$ & $\begin{array}{l}\text { cailloux } \\
\text { pierres } \\
\text { mousses }\end{array}$ & $\begin{array}{c}30.8 .76 \\
2.9 .76 \\
. "\end{array}$ \\
\hline $\begin{array}{l}46 \text { Neste de Louron } \\
47 \text { Neste de Lavedan } \\
48\end{array}$ & $\begin{array}{l}960 \mathrm{~m} \\
860 \mathrm{~m} \\
n\end{array}$ & $\begin{array}{l}\text { vif } \\
\text { rapide }\end{array}$ & $\begin{array}{l}\text { pierres } \\
\text { pierres } \\
\text { mousses }\end{array}$ & $\begin{array}{c}16.9 .76 \\
4.9 .76 \\
.7\end{array}$ \\
\hline 49 r. d'Espiaube & $825 \mathrm{~m}$ & vif & pierres & 5.9 .75 \\
\hline 50 & $"$ & & mousses & 5.9 .75 \\
\hline $\begin{array}{l}51 \text { Neste d'Aure (à Vielle) } \\
52\end{array}$ & $\stackrel{790 \mathrm{~m}}{\prime \prime}$ & rapide & $\begin{array}{l}\text { pierres } \\
\text { mousses }\end{array}$ & $\begin{array}{l}19.9 .76 \\
" 1\end{array}$ \\
\hline $\begin{array}{l}53 \text { La Mousquère, aval Bourisp } \\
54\end{array}$ & $790 \mathrm{~m}$ & $\begin{array}{l}\text { vif } \\
\text { vif }\end{array}$ & $\begin{array}{l}\text { pierres } \\
\text { pierres }\end{array}$ & $\begin{array}{l}25.7 .76 \\
26.8 .76\end{array}$ \\
\hline
\end{tabular}


Tableau I. (suite)

\begin{tabular}{|c|c|c|c|c|}
\hline COURS D'EAU & ALTITUDE & COURANT & SUBSTRAT & DATE \\
\hline $55 \mathrm{r}$. d'Aspin & $775 \mathrm{~m}$ & vif & pierres & $\begin{array}{c}26.8 .76 \\
" 1\end{array}$ \\
\hline $\begin{array}{l}57 \text { Le Lastie } \\
58\end{array}$ & $757 \mathrm{~m}$ & rapide & $\begin{array}{l}\text { pierres } \\
\text { mousses }\end{array}$ & 6.9 .75 \\
\hline 59 Neste d'Aure à Grézian & $746 \mathrm{~m}$ & vif & pierres & 24.8 .75 \\
\hline 60 Neste de Louron & $895 \mathrm{~m}$ & vif & pierres & 21.9 .75 \\
\hline 61 & " & vif & mousses & $"$ \\
\hline $\begin{array}{l}62 \mathrm{r} \text {, de Beyrède } \\
63\end{array}$ & ${ }^{666 \mathrm{~m}}$ & $\begin{array}{l}\text { vif } \\
\text { vif }\end{array}$ & $\begin{array}{l}\text { pierres } \\
\text { mousses }\end{array}$ & 4.9 .75 \\
\hline $\begin{array}{l}64 \text { r. d'Ardengost } \\
65\end{array}$ & $\begin{array}{c}665 \mathrm{~m} \\
"\end{array}$ & $\begin{array}{l}\text { vif } \\
\text { vif }\end{array}$ & $\begin{array}{l}\text { pierres } \\
\text { mousses }\end{array}$ & 4.9 .75 \\
\hline $\begin{array}{l}66 \text { L'Arrieu (à Camous) } \\
67\end{array}$ & $\stackrel{670 \mathrm{~m}}{\prime \prime}$ & $\begin{array}{l}\text { vif } \\
\text { vif }\end{array}$ & $\begin{array}{l}\text { pierres } \\
\text { mousses }\end{array}$ & 3.8.76 \\
\hline $\begin{array}{l}68 \text { г. de Beyrède } \\
69\end{array}$ & $1180 \mathrm{~m}$ & $\begin{array}{l}\text { vif } \\
\text { vif }\end{array}$ & $\begin{array}{l}\text { pierres } \\
\text { mousses }\end{array}$ & 28.8 .76 \\
\hline 70 Neste d'Aure à Lortet & $535 \mathrm{~m}$ & vif & pierres & 27.8 .76 \\
\hline 71 & $535 \mathrm{~m}$ & vif & mousses & "' \\
\hline 72 & " & vif & mous. (bord) & $"$ \\
\hline 73 Neste d'Aure à Bizous & $490 \mathrm{~m}$ & lent & pierres & 11.8 .76 \\
\hline $\begin{array}{l}74 \text { Neste du Nistos } \\
75\end{array}$ & $\begin{array}{c}866 \mathrm{~m} \\
n\end{array}$ & $\begin{array}{l}\text { vif } \\
\text { vif }\end{array}$ & $\begin{array}{l}\text { pierres } \\
\text { mousses }\end{array}$ & 25.8 .76 \\
\hline $76 \quad " \prime$ & $526 \mathrm{~m}$ & vif & pierres & $"$ \\
\hline 77 & " & vif & mousses & " \\
\hline 78 & $455 \mathrm{~m}$ & lent & pierres & 11.8 .77 \\
\hline 79 & " & vif & pierres & $"$ \\
\hline 80 L'Arros & $422 \mathrm{~m}$ & vif & pierres & 26.8 .76 \\
\hline 81 & $"$ & vif & mousses & $"$ \\
\hline 82 L'Esqueda & $407 \mathrm{~m}$ & lent & pierres & 28.8 .76 \\
\hline 83 & " & lent & mousses & $"$ \\
\hline 84 & $"$ & vif. & mous. (casc) & $"$ \\
\hline 85 & $"$ & vif & pierres & $"$ \\
\hline 86 r. de Matau & $430 \mathrm{~m}$ & vif & cailloux & 26.8 .76 \\
\hline 87 & ," & vif & mousses & $"$ \\
\hline $88 \mathrm{r}$. de l'Ayguette & $440 \mathrm{~m}$ & lent & pierres & 26.8 .76 \\
\hline 89 & $"$ & lent & mousses & $"$ \\
\hline 90 Le Gers & $275 \mathrm{~m}$ & vif & cailloux & 25.8 .77 \\
\hline 91 La Baïsole & $252 \mathrm{~m}$ & lent & pierres & $"$ \\
\hline 92 " & & lent & mousses & $"$ \\
\hline 93 La Baïse & & lent & cailloux & 25.8 .77 \\
\hline 94 Le Boues & $301 \mathrm{~m}$ & lent & cailloux & 25.8 .77 \\
\hline 95 L'Arros & $212 \mathrm{~m}$ & lent & cailloux & 25.8 .77 \\
\hline 96 & & vif & mousses & $"$ \\
\hline 97 Le Soubirou & $1580 \mathrm{~m}$ & vif & cailloux & 12.8 .76 \\
\hline 98 L'Artigou & $1395 \mathrm{~m}$ & rapide & cailloux & 12.8 .76 \\
\hline 99 Le Camoudiet & $1440 \mathrm{~m}$ & rapide & mousses & 12.8 .76 \\
\hline 100 & & & mous, hygr. & $"$ \\
\hline 101 & $1300 \mathrm{~m}$ & vif & cailloux & $"$ \\
\hline 102 & - & vif & mousses & " \\
\hline 103 L'Hourc & $1250 \mathrm{~m}$ & rapide & mousses & 12.8 .76 \\
\hline 104 & $1150 \mathrm{~m}$ & rapide & mousses & " \\
\hline 105 & & lent & mousses & $"$ \\
\hline $\begin{array}{l}106 \text { r. de Morère } \\
107\end{array}$ & $1139 \mathrm{~m}$ & rapide & pierres & ${ }^{12.8 .76}$ \\
\hline $108 \quad$ " & $1080 \mathrm{~m}$ & vif & $\begin{array}{l}\text { mousses } \\
\text { pierres }\end{array}$ & 12.7 .76 \\
\hline
\end{tabular}




\subsection{1. - Haute vallée de l'Adour (stations 97 à 108)}

Elle est limitée au nord et à l'est par des crêtes ne dépassant pas $1900 \mathrm{~m}$; au sud et à l'ouest, les sommets sont plus élevés $(2500$ à $2830 \mathrm{~m})$. Le versant nord de la vallée est constitué notamment de schistes permo-triasiques; calcaires et schistes dinantiens dominent à l'est, tandis que le massif de l'Arbizon, au sud, est formé par le granite de la zone axiale.

L'Adour résulte de la confluence, au pied du col d'Aspin, de ruisseaux très divers. Camoudiet (st. 99 à 102) et Artigou (st. 98) prennent leur source res. pectivement à 2250 et $2700 \mathrm{~m}$ d'altitude, et le régime des eaux est de type nival de transition. La conductivité des eaux varie entre 137 (st. 98) et 210 $\mu$ mhos $/ \mathrm{cm}$ (st. 99 à 102) en étè.

Les ruisseaux qui descendent du col d'Aspin et du versant nord de la vallée ( $s$. 103 à 108) ont un régime de type pluvio-nival et coulent dans une hêtraiesapinière. La conductivité au niveau des stations étudiées varie entre 80 et $175 \mu \mathrm{mhos} / \mathrm{cm}$.

\section{J.2. - Les Baronnies (stations 80 à 89)}

Entre le plateau de Lannemezan et le cône de Cieutat-Orignac, les Baronnies sont une région de hautes collines prépyrénéennes qui prolongent vers l'est les collines de Bigorre. Elles sont draînées par l'Arros (qui traverse ensuite les Coteaux de Gascogne et se jette dans l'Adour) et ses affluents, Esquéda, Ayguette et Matau. Ces ruisseaux prennent leur source vers $1200 \mathrm{~m}$ d'altitude et coulent sur calcaire (conductivité : 268 à $475 \mu \mathrm{mhos} / \mathrm{cm}$ ). Le régime des eaux est de type pluvio-nival ou pluvial. Les eaux se réchauffent rapidement au printemps (12 à $13^{\circ}$ $\mathrm{C}$ en avril), mais ne dépassent guère $15^{\circ} \mathrm{C}$ en été.

\section{1.t.3. - Les Coteaux de Gascogne (stations 90 à 96)}

Les rivières des Coteaux de Gascogne appartiennent à deux grands bassins hydrographiques. Les unes (Gers, Baïse, Baïsole,...) sont des affluents de la Garonne (comme la Neste d'Aure). Les autres, dont le Bouès, affluent de l'Arros, se jettent dans l'Adour. Elles prennent leur source sur le plateau de Lannemezan, à $600 \mathrm{~m}$ d'altitude. Ce plateau reçoit plus de $900 \mathrm{~mm}$ d'eau par an, mais sa surface est insuffisante pour alimenter la dizaine de rivières qui prennent leur source côte à côte. Elles se partagent, en été, les quelques $\mathrm{m}^{3 /} \mathrm{sec}$. qu'un canal creusé au $19^{e}$ siècle leur apporte à partir de la Neste d'Aure.

Les rivières gasconnes coulent dans des molasses. Ce sont des rivières à faible courant en dehors des périodes de crue. le régime des eaux est de type pluviothermique gascon ( $R$. Lambert, 1975), avec des crues fréquentes au printemps, plus rares en automne, et des étiages prononcés en été, rappelant les rivières méditerranéennes.

Coulant d'abord sur des terrains siliceux, les rivières gasconnes s'enrichissent progressivement en bicarbonates (conductivité de l'ordre de 130 à 140 $\mu$ mhosicm en été, au niveau des stations étudiées).

\section{2. - Les Hydracariens des eaux courantes des Pyrénées centrales et de la Gascogne (tableau II)}

86 espèces (Hydrachnellae et Porohalacaridae) ont été recueillies. Trois espèces du gen re Lebertia n'ont pu être déterminées avec précision dans l'état actuel de la systématique.

Sur le tableau II sont regroupées dans la colonne « Haute vallée d'Aure » les stations 1 à 37, situées au-delà de $1100 \mathrm{~m}$ d'altitude. la * Moyenne vallée d'Aure * rassemble les stations 38 à 69 et 74 à 77 , entre 600 et $1100 \mathrm{~m}$; la "Basse vallée d'Aure ", au-dessous de $600 \mathrm{~m}$, les stations 70 à 73,78 et 79 .

Les prélèvement s ont été effectués au filet de Surber sur les fonds de galets. Les mousses ont été lavées sous un jet d'eau.

\section{2. - Les paramètres des stations}

22 paramètres ont été pris en compte pour caractériser chacune des 108 stations. 9 d'entre eux sont habituellement considérés comme des facteurs écologiques susceptibles d'influencer directement la répartition de la faune aquatique :

- température

- conductivité

- alcalinité

$-\mathrm{pH}$

- oxygène dissous

- vitesse du courant

- granulométrie du substrat

- abondance des Bryophytes

- nature des Bryophytes 
Tableau II. Les Hydracariens des Pyrénées centrales et des Coteaux de Gascogne

\begin{tabular}{|c|c|c|c|c|c|c|}
\hline & 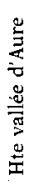 & 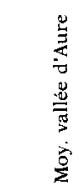 & 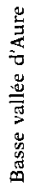 & 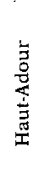 & 菢 & $\begin{array}{l}\stackrel{0}{0} \\
\stackrel{0}{8} \\
\dot{6} \\
0\end{array}$ \\
\hline V01 Hydrovolzia cancellata Walt. 1906 & + & & & & & \\
\hline v02 Hydrovolzia placophora (Monti 1905) & + & & & & & \\
\hline V03 Protzia eximia (Protz 1896) & & + & & + & + & \\
\hline V04 Protzia invalvaris Piers. 1898 & + & + & + & + & + & + \\
\hline V06 Protzia rotunda Walt. 1908 & & + & & + & + & \\
\hline V05 Protzia squamosa Walt. 1908 & & + & & & + & \\
\hline * Wandesia thori Schechtel 1912 & + & & & & & . \\
\hline V07 Panisopsis curvifrons (Walt. 1907) & + & + & & + & & \\
\hline V08 Panisopsis thori (Walt. 1907) & + & + & & & & \\
\hline V11 Trichothyas petrophila (Mich. 1895) & & + & & & & \\
\hline V09 Panisus michaeli Koen. 1896 & + & + & & + & & \\
\hline V10 Panisus torrenticolus Piers. 1898 & & + & & + & + & \\
\hline V12 Hydryphantes pyrenaicus E. Ang. (nom. nud.) & + & & & & & \\
\hline V15 Sperchon brevirostris (Koen. 1895) & + & + & & & & \\
\hline V18 Sperchon glandulosus (Koen. 1886) & + & + & & + & & \\
\hline V16 Sperchon denticulatus (Koen. 1895) & + & + & + & + & + & + \\
\hline V17 Sperchon denticulatus hibernicus (Halb. 1944) & + & & & & & \\
\hline V14 Sperchon clupeifer (Piers, 1896) & & + & + & + & + & + \\
\hline V13 Sperchon hispidus (Koen. 1895) & + & + & + & & & \\
\hline V82 Sperchon papillosus (Thor 1901) & + & & & & & \\
\hline V77 Sperchonopsis verrucosa (Protz 1896) & + & + & + & + & + & + \\
\hline V70 Lebertia fimbriata Thor 1899 & + & + & & + & + & + \\
\hline V72 Lebertia maglioi (Thor 1907) & & + & & + & + & + \\
\hline V80 Lebertia sparcicapillata (Thor 1905) & & + & & & & \\
\hline V66 Lebertia insignis (Neumann 1880) & & + & + & & & + \\
\hline V65 Lebertia porosa (Thor 1900) & & + & + & & + & + \\
\hline V75 Lebertio glabra Thor 1897 & & & + & & + & \\
\hline V67 Lebertia lusitanica Lundb. 1956 & + & & & & & \\
\hline V69 Lebertia salebrosa (Koen. 1908) & + & & & & & \\
\hline V68 Lebertia zschokkei (Koen. 1902) & + & + & & & & \\
\hline V74 Lebertia dubia (Thor 1899) & + & & & + & & \\
\hline V73 Lebertia sefvet (Walt. 1911) & + & & & & & \\
\hline V71 Lebertia stigmatifera (Thor 1900) & & + & & & & \\
\hline V42 Lebertia sp. A & & & & & & + \\
\hline V76 Lebertia sp. B & & & & & & + \\
\hline V81 Lebertia sp. C & + & + & & & & \\
\hline V19 Frontipoda musculus (Müll. 1776) & & & + & & & \\
\hline V20 Torrenticola anomala (Koch 1837) & & & + & & + & + \\
\hline V26 Torrenticola brevirostris (Halb. 1911) & & & & & & + \\
\hline V21 Torrenticola fagei (E. Ang. 1949) & + & & & & & \\
\hline V23 Torrenticola madritensis (Viets 1930) & & + & + & + & + & + \\
\hline V27 Torrenticola remyi (E. Ang. 1954) & & & & & & + \\
\hline V22 Torrenticola similis (Viets 1939) & + & + & + & + & + & + \\
\hline V25 Torrenticola stadleri (Walt 1924) & & & & & & + \\
\hline V24 Torrenticola amplexa (Koen. 1908) & & & + & & & + \\
\hline V78 Pseudotorrenticola rhynchota Walt. 1906 & & + & + & & + & \\
\hline V29 Hygrobates calliger Piers. 1896 & & + & + & + & + & + \\
\hline V79 Hygrobates fluviatilis (Ström 1768) & & & & & + & + \\
\hline V28 Hygrobates nigromaculatus Leb. 1879 & + & & + & & & \\
\hline
\end{tabular}


Tableau II. (suite)

\begin{tabular}{|c|c|c|c|c|c|c|}
\hline & 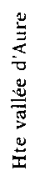 & 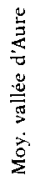 & 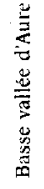 & 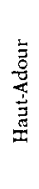 & 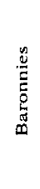 & $\begin{array}{l}\mathscr{E}_{0} \\
\text { ర్ } \\
\text { S }\end{array}$ \\
\hline V30 Hygrobates (Rivob.) norvegicus (Thor 1897) & + & & & & & \\
\hline V31 Atractides acutirostris (Mot. et Ang. 1927) & & & & & & + \\
\hline V4I Atractides fluviatilis (Szalay 1929) & & + & & & & \\
\hline V45 Atractides fonticolus (Viets 1920) & & + & & + & & \\
\hline V35 Atractídes gibberipalpis Piers, 1898 & + & + & + & + & + & \\
\hline V44 Atractides latipalpis (Mot. et Tan. 1946) & + & + & & & & \\
\hline V33 Atractides loricatus Piers. 1898 & + & & & & & + \\
\hline V36 Atractides lunipes Lundb. 1956 & + & & & & & \\
\hline V39 Atractides nodipalpis (Thor 1899 ) & & + & + & + & + & + \\
\hline V38 Atractides nodipalpis pennatus (Viets 1920 ) & & + & + & & & + \\
\hline V37 Atractides pumilus (Szalay 1916) & & + & & & & \\
\hline V34 Atractides spinipes (Koch 1837) & & + & & & & \\
\hline V46 Atractides subasper Koen. 1902 & & & & & & + \\
\hline V32 Atractides tener (Thor 1899 ) & & & + & + & + & \\
\hline V40 Atractides vaginalis (Koen. 1905) & + & + & & & & \\
\hline V43 Atractides octoporus (Piers. 1904) & & & + & & + & \\
\hline V48 Feltria armata Koen. 1902 & + & + & + & + & + & \\
\hline V52 Feltria bispinosa Ang. 1950 & + & + & & + & & \\
\hline V47 Feltria minuta Koen. 1892 & + & & & & & \\
\hline V50 Feltria quadrispinosa E. Ang. (nom. nud.) & + & & & & & \\
\hline V49 Feltria rouxi Walt. 1907 & & & & & + & \\
\hline V51 Feltria rubra (Piers. 1898) & + & + & & & & \\
\hline * Nautarachna crassa (Koen. 1908) & & & + & & & \\
\hline V53 Woolastookia rotundifrons (Viets 1922) & & & & + & + & + \\
\hline V54 Ljania bipapillata Thor 1898 & & & & + & + & \\
\hline V61 Aturus crinitus Thor 1902 & & & + & & + & \\
\hline V60 Aturus elongatus processiger Lundb 1956 & + & + & & + & + & \\
\hline V55 Aturus prenanti E. Ang. 1965 & & + & & + & & \\
\hline V58 Aturus protzi Piers. 1901 & & & & & + & \\
\hline V56 Aturus scaber Kram. 1875 & & + & + & + & + & + \\
\hline V57 Aturus spatulifer Piers. 1904 & + & + & & & & \\
\hline V59 Aturus villosus Mot. et Soar. 1939 & & + & & & & \\
\hline V62 Kongsbergia clypeata Szalay 1945 & & + & & & & \\
\hline V63 Kongsbergia matema Thor 1899 & & + & & + & + & \\
\hline V64 Kongsbergia nuttneri Walt. 1930 & & & + & & & \\
\hline * Mideopsis orbicularis (Müll. 1776 & & & + & & & \\
\hline \multicolumn{7}{|l|}{ Porohalacaridae } \\
\hline * Soldanellonyx chappuisi Walt. 1917 & + & & & & & \\
\hline
\end{tabular}

* Wandesia thori, Nautarachna crassa, Mideopsis orbicularis et Soldanellonyx chappuisi recueillis seulement lors d'études de dérive (Gazagnes 1983 et V. Bougenec, S. Meurgues \& C. Sourzac, 1984) ne figurent pas dans les 108 stations prospectées.

Les indices affectés à chaque espèce correspondent au codage utilisé dans les analyses factorielles des correspondances. 
Les teneurs de l'eau en oxygène correspondent pratiquement a la saturation dans l'ensemble des stations ; nous n'avons donc pas retenu ce paramètre dans les analyses statistiques, car il ne permet pas de différencier les stations les unes des autres. Les données retenues sont celles de la fin de l'été ou début de l'automne (période d'étiage aux conductivités et températures maximales sur l'ensemble des stations et également aux peuplements les plus diversifiés).

13 paramètres rendent compte du bassin versant ou de la morphométrie du cours d'eau :

- altitude de la source

- régime des eaux

- numéro d'ordre du ruisseau
- surface du bassin versant

- orientation de la vallée

- pente moyenne en amont de la station

- nature géologique du bassin versant

- \% boisé du bassin versant

- action humaine

- altitude de la station

- distance source-station

- pente au niveau de la station

- largeur du cours d'eau

L'ensemble de ces 13 paramètres constitue un complexe stationnel dont nous devons rechercher les relations avec les facteurs écologiques soupçonnés ou réels.

Tableaı III. Classes des paramètres et indices biospécifiques

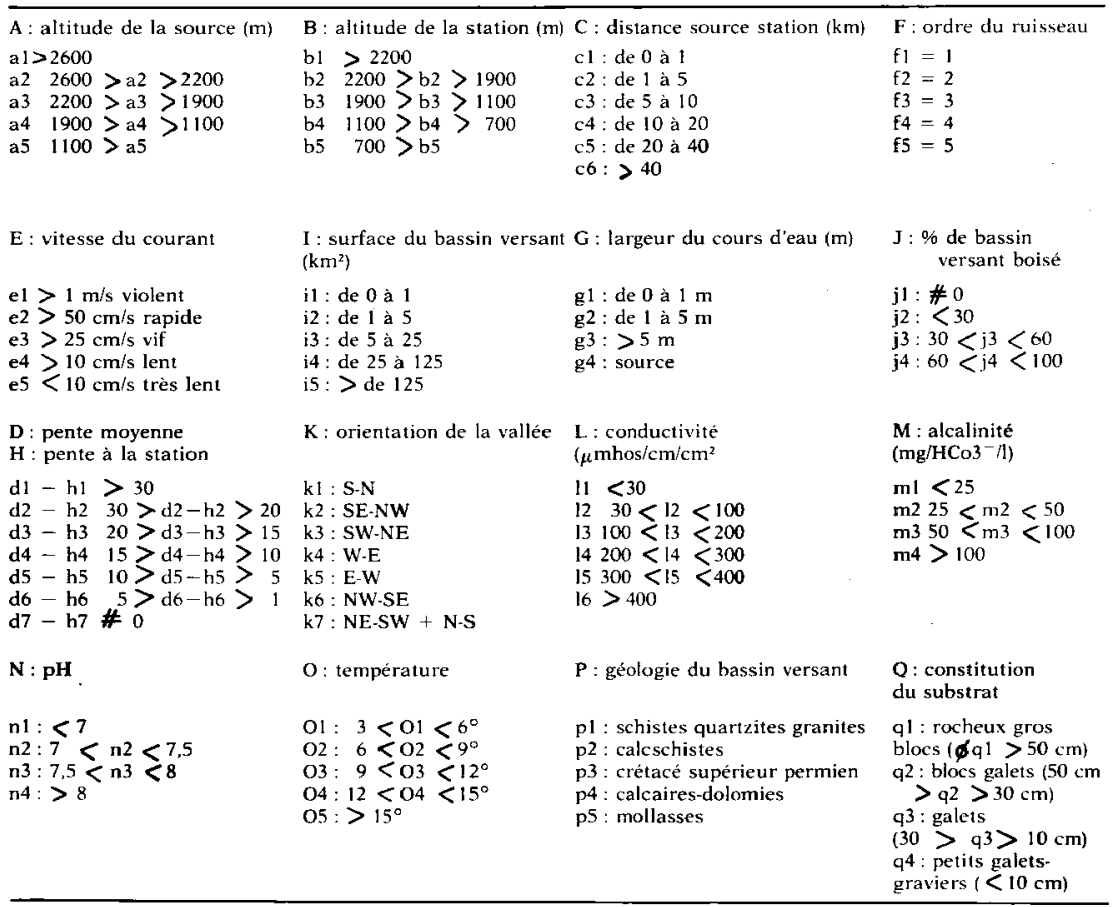


Tableau III. (suite)

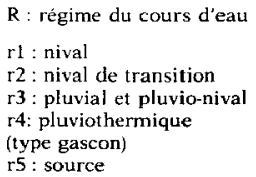

Si : Simpson, indice de dominance

sil: de 0 à 0,1

si 2 : de $0, \mathrm{j}$ à 0,2

si3 : de 0,2 à 0,3

si4 : de 0,3 à 0,4

si5: de 0,4 à 0,5
$S$ : interventions humaines

s1: pacage

$s 2$ : pacage + barrage

s3: pacage + barrage

+ agriculture

s4 : pacage + barrage

+ agriculture

+ agglomérations

Wi : indice de Fisher

wil : de 0 à 1,9

wi2: de 2 à 3,9

wi3 : de 4 à 5

wi4: de 5 à 6

wi5 : $>6$
I : présence de Bryophytes

11: absentes

t2: présentes

3 : abondantes

$U$ : types de Bryophytes

u1: absence

u2 : brvacées

dominantes

u3: hypnacées

dominantes

u4: hypnacées
E/G : nbre d'espèces/

nbre de genres

e/g 1: de 1 à 1,2

e/g2: de 1,2 à 1,4

e/g3 : de 1,4 à 1,6

e/g4 : de 1,6 à 1,8

e/g5 : de 1,8 à 2

e/g6: $>2$
21 paramètres (l'oxygène dissous n'étant pas pris en compte) comportant au total 105 classes (tableau III) constituent donc un premier ensemble de variables, et les 108 stations un second ensemble, dans une série d'analyses factorielles des correspondances qui ont été réalisées.

\section{1. - Analyse factorielle des correspondances paramètres-stations (fig. 2 à 4)}

Les 3 premiers axes rendent compte de $23,6 \%$ de l'inertie totale du nuage de points. 3 groupes de stations et de paramètres s'opposent sur les axes I et II : - les sources d'altitude, positives sur l'axe I et négatives sur l'axe II (stations 1, 7, 8, 9, 10, 12, 17, 21, 24 à 26, 29, 30, 36 et 98). Elles sont liées aux paramètres $\mathrm{U}_{3}$ (Hypnacées dominantes), $\mathrm{B}_{1}$ et $\mathrm{B}_{3}$ (altitudes élevées), $M_{1}$ (alcalinité inférieure à $25 \mathrm{mg} / \mathrm{l}$ ). Les sources limnocrènes s'isolent de cet ensemble par leur pente $\left(D_{4}\right)$ et leur courant $\left(E_{5}\right)$ pratiquement nuls.

- les stations des Coteaux de Gascogne (90 à 96), en position négative sur les axes I et II avec les paramètres $B_{5}$ (altitude inférieure à $700 \mathrm{~m}$ ), $D_{6}$ (pente inférieure à $5 \%$ ), $P_{5}$ (terrains mollassiques), $C_{5}$ (distance source-station entre 20 et $40 \mathrm{~km}$ ) et $\mathrm{O}_{5}$ (température estivales supérieures a $15^{\circ} \mathrm{C}$ ).

- les stations des Baronnies ( 80 à 89 ), positives sur l'axe I, mais d'un faible poids sur l'axe II, avec les paramètres $A_{5}$ (altitude de la source inférieure à $1100 \mathrm{~m}$ ), $\mathrm{D}_{5}$ (pente entre 5 et $10 \%$ ), $\mathrm{O}_{4}$ (tempé- ratures estivales entre 12 et $15^{\circ} \mathrm{C}$ ), $\mathrm{C}_{3}$ (distance source-station entre 5 et $10 \mathrm{~km}$ ), $\mathbf{M}_{4}$ (alcalinité supérieure à $100 \mathrm{mg} / \mathrm{l}$ ).

Entre les sources de haute altitude et les Baronnies sont situées les stations de la haute et moyenne vallée d'Aure (1 à 69, sauf les sources), du haut Adour et du haut Nistos $(74,75,97,108)$, positives sur l'axe I. Les stations de la basse vallée d'Aure et du Nistos inférieur (70 à 73,76 à 79 ) figurent entre les Baronnies et les Coteaux de Gascogne.

Sur l'axe III s'opposent (fig. 4) :

- les stations des Baronnies, négatives sur l'axe, avec les paramètres $\mathrm{M}_{4}$ (alcalinité supérieure à $100 \mathrm{mg} / \mathrm{l}), \mathrm{N}_{4}\left(\mathrm{pH}\right.$ supérieur à 8 ) et $\mathrm{R}_{3}$ (régime des eaux pluvial ou pluvio-nival),

- à un groupe de stations de moyenne altitude positives sur l'axe, - de la Neste d'Aure et de ses affluents (st. 42 à 48 et 75 ), liées aux paramétres $\mathrm{D}_{4}$ (pente entre 10 et $15 \%$ ), $A_{2}$ (altitude de la source entre 2200 et $2600 \mathrm{~m}$ ), $\mathrm{C}_{3}$ et $\mathrm{C}_{4}$ (distance solifetstation entre 5 et $20 \mathrm{~km}$ ), et $R_{2}$ (Régime nival de transition).

L'axe I représente un facteur " physionomieg du cours d'eau ", lié à un ensemble de paranètres qui varient régulièrement de l'amont vers l'aval : alt itude et pente décroissantes, numéro d'ordre du cours d'eau, distance à la source, largeur du lit, températures estivales et action humaine croissants. A l'extrémité positive de l'axe', les sources de haute altitude ; à l'extrémité négative, les rivièrés gasconnes. 


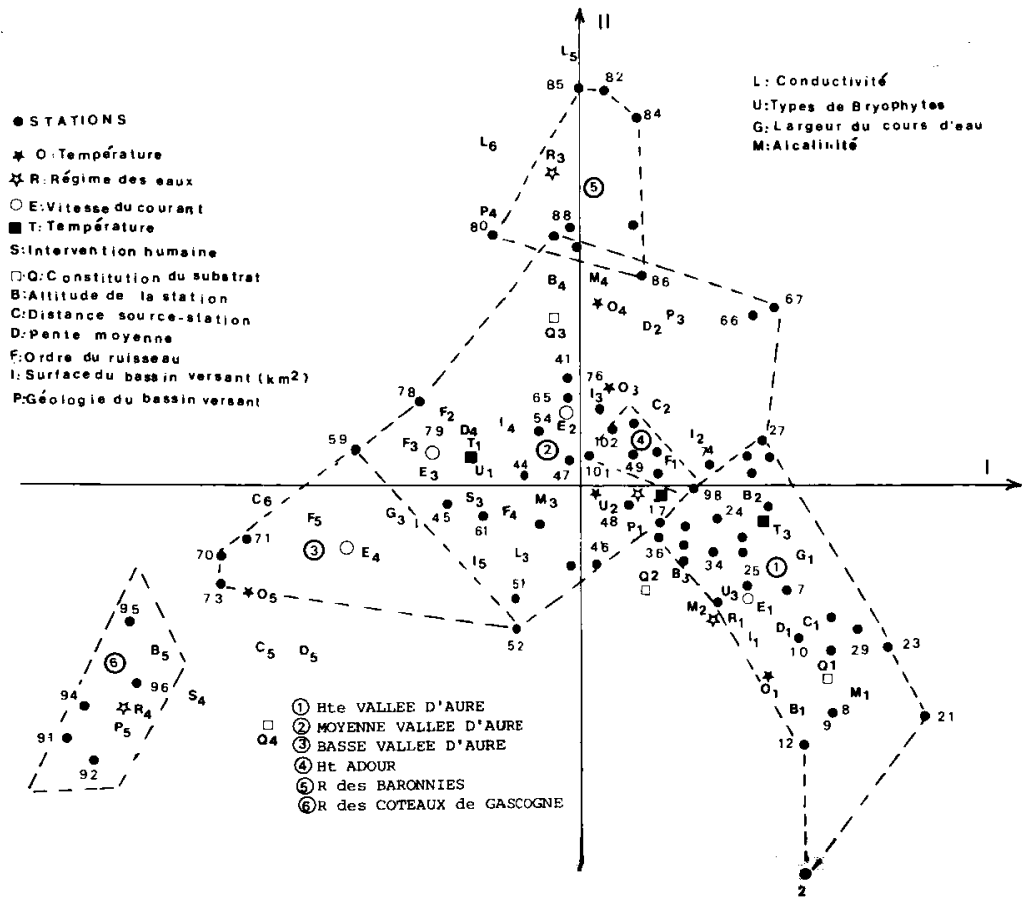

Fig. 2. Analyse factorielle des correspondances stations-paramètres. Axes I et II.

Sur un même cours d'eau, l'axe I représente finalemerit'l'évolution progressive des paramètres cités lé lóng d’un profil d'équilibre.

Gaxe II représente un facteur de spécialisation opposant des stations aux caractéristiques moyennes, - les Baronnies, - à des stations aux conditions extrêmes : rivières gasconnes et sources de haute altitude, régime des eaux pluviothermique ou trival:

L'axe III traduit à la fois les composantes chimiques et le régime des eaux. Il oppose les stations des Baronnies, à régime pluvial ou pluvio-nival et minéralisation élevée à des stations à régime nival de transition et minéralisation plus faible. Il traduit en fait l'opposition entre le piémont pyrénéen et les massifs centraux.
Une analyse en composantes principales confirme les résultats de l'analyse factorielle des correspondances.

\section{2. - Hérarchisation des paramètres sur les 2 et 5 premiers axes (fig. 5 et 6 ).}

Un certain nombre de paramètres sont évidemment liés entre eux. Une hiérarchisation des paramètres, - tirée de l'analyse factorielle des correspondances, - fait apparaittre leur niveau de liaison.

Tant sur 2 que sur 5 axes, pH, conductivité et alcalinité sont étroitement corrélés, de sorte que la minéralisation de l'eau peut être correctement exprimée avec un seul de ces trois paramètres. 

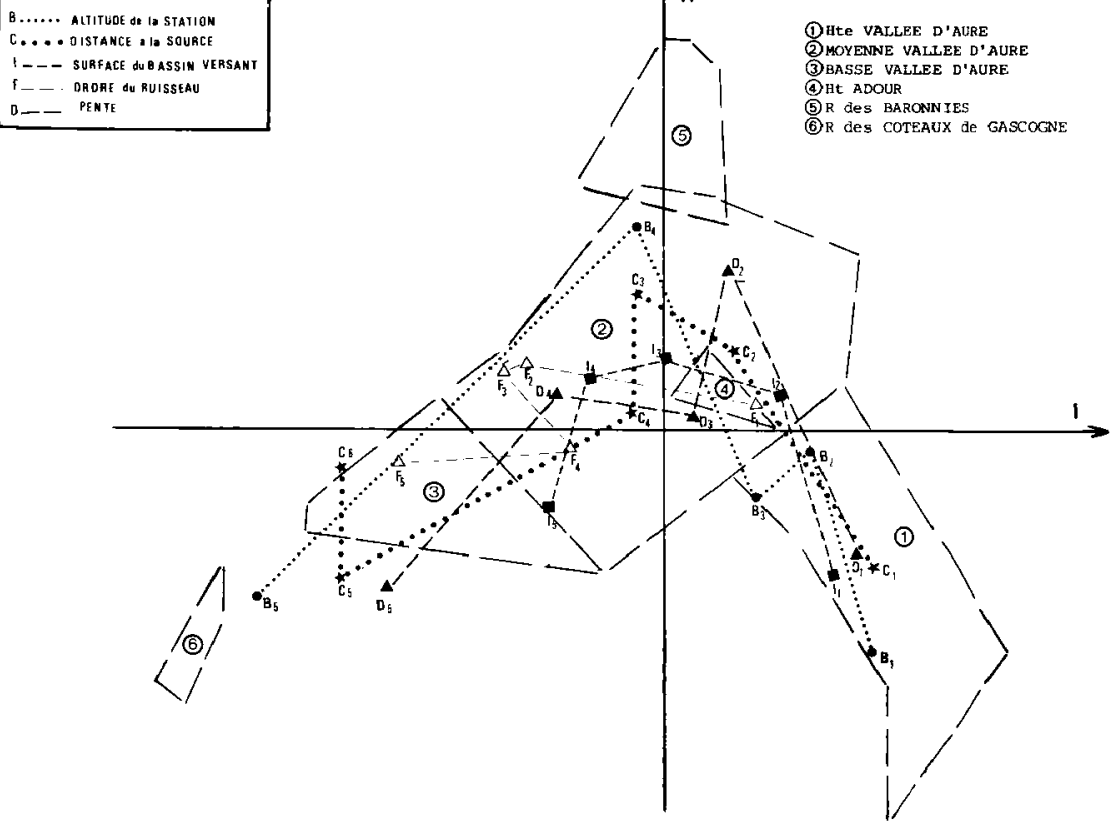

Fig. 3. Analyse factorielle des correspondances stations-paramètres. Evolution de quelques paramètres essentiels sur les axes I et II.

D'autres paramètres sont également corrélés, entre eux et avec les précédents, à des niveaux élevés (supérieurs à 0,8 ): distance source stationtempérature, pente-diamètre des galets-vitesse du courant, surface du bassin versant-numéro d'ordre du cours d'eau,... Tous ces paramètres reflètent la physionomie du cours d'eau de l'amont vers l'aval.

La liaison entre l'altitude de la source et le régime des eaux apparaît, sur les 2 premiers axes (niveau 0,8 ), mais elle est égale à 0,65 seulement sur 5 axes. Quant aux mousses, elles sont relativement indépendantes des autres paramètres (niveau de liaison infé. rieur à 0,7 sur 2 axes, inférieur à 0,6 sur 5 axes).

Le niveau de liaison entre la plupart des paramètres est tel qu'un nombre réduit d'entre eux suffit probablement à rendre compte du complexe stationnel. C'est ce que confirme l'analyse canonique.

\section{3. - Analyse canonique sur les paramètres quantitatifs (fig. $7 \mathrm{~A}$ et $7 \mathrm{~B}$ )}

Cette analyse a porté sur 2 ensembles de paramètres, caractérisant 10 stations à substrat de mousses et 10 stations à substrats de galets de la vallée d'Aure (jusqu'à $2200 \mathrm{~m}$ ), des Baronnies et des Coteaux de Gascogne. Elle a pour but de vérifier si une station peut être caractérisée, écologiquement, par des seuls paramètres topographiques. Nous avons ajouté aux analyses les indices biospécifiques calculés pour le peuplement de chacune des 20 stations. 


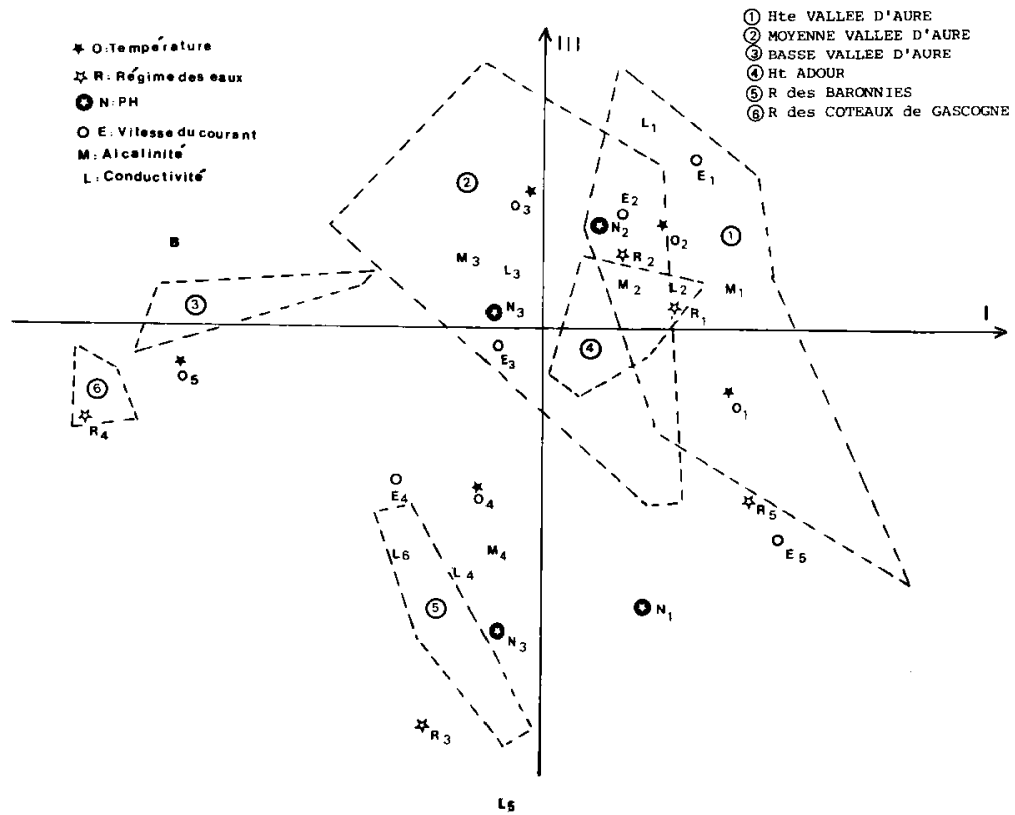

Fig. 4. Analyse factorielle des correspondances stations-paramètres. Axes 1 et III.

- Ensemble des paramètres topographiques: altitude de la source altitude de la station distance source-station surface du bassin versant largeur du cours d'eau pente de la station

- Ensemble des paramètres écologiques et biospécifiques: conductivité température vitesse du courant indice de Fisher indice de Simpson rapport Espèces/Genres

Deux coefficients de corrélation cảnonique sont hautement significatifs et indiquent que les deux ensembles de paramètres sont fortement corrélés.
La liaison entre les ensembles est due essentiellement :

- à la pente (test de contribution : 0,928 ) et à la vitesse du courant $(0.86)$;

- accessoirement, à la distance source-station $(0,24)$ et à l'indice de Fisher $(0,38)$.

Equation canonique (fig. $7 \mathrm{~A})$ :

$$
\begin{aligned}
\mathrm{U}_{1}= & 0,92 \text { (pente) }+0,24 \text { (distance source-station) } \\
\mathrm{V}_{1}= & 0,86 \text { (vitesse du courant) }+0,38 \text { (indice de } \\
& \text { Fisher) }+0,32 \text { (température) }
\end{aligned}
$$

Si on élimine l'action des facteurs $U_{1}$ et $V_{1}$, une seconde liaison entre les deux ensembles de paramètres est due à l'altitude de la station (test de contribution : 0,56), la surface du bassin versant ($0,55)$, l'altitude de la source $(0,47)$ et la température $(-0,80)$. 


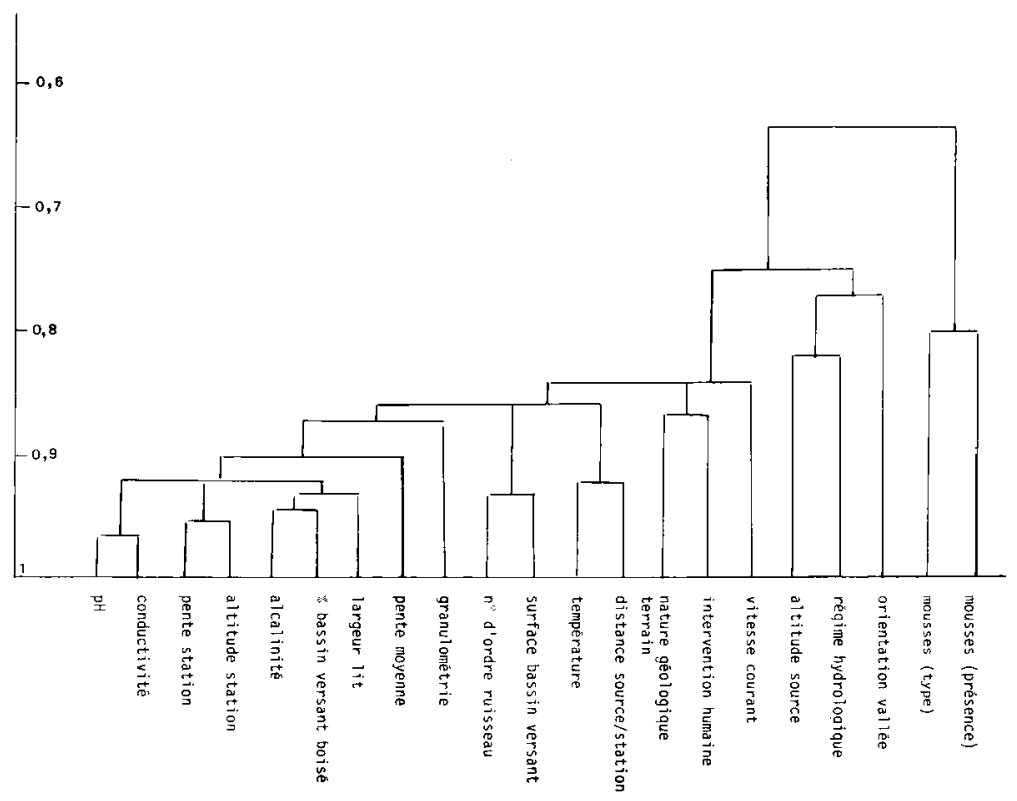

Fig. 5. Hiérarchisation des paramètres sur les deux premiers axes.

Equations canoniques (fig. $7 \mathrm{~B}$ ) :

$\mathrm{U}_{2}=0,56$ (altitude de la station) $-0,55$ (surface du bassin versant) $+0,47$ (altitude de la source).

$V_{2}=0,80$ (température).

Les 20 stations dont les paramètres ont permis l'analyse canonique se disposent à peu près sur une droite, par rapport aux axes $\mathrm{U}_{1}$ et $\mathrm{V}_{1}, \mathrm{U}_{2}$ et $\mathrm{V}_{2}$.

En principe, les paramètres topographiques, pente et altitude de la station, altitude de la source, surface du bassin versant, accessoirement distance source-station, devraient rendre compte des paramètres écologiques fondamentaux, vitesse du courant et température, et de la diversité du peuplement (indice de Fisher). Mais il ne faut pas oublier que les paramètres non quantifiables ne peuvent être pris en compte dans une analyse canonique. C'est notamment le cas du régime des eaux, bien que celui-ci présente une certaine corrélation avec l'altitude de la source.

\section{3. - Les associations d'Hydracariens et les relations espèces- paramètres des milieux}

L'analyse factorielle des correspondances a été réalisée, comme pour les paramètres physicochimiques et topographiques, sur les récoltes de fin d'été et d'automne. C'est à cette époque que la diversité maximale des taxocoenoses est atteinte et que la majeure partie des espèces est présente à un stade ou à un autre de son développement. 


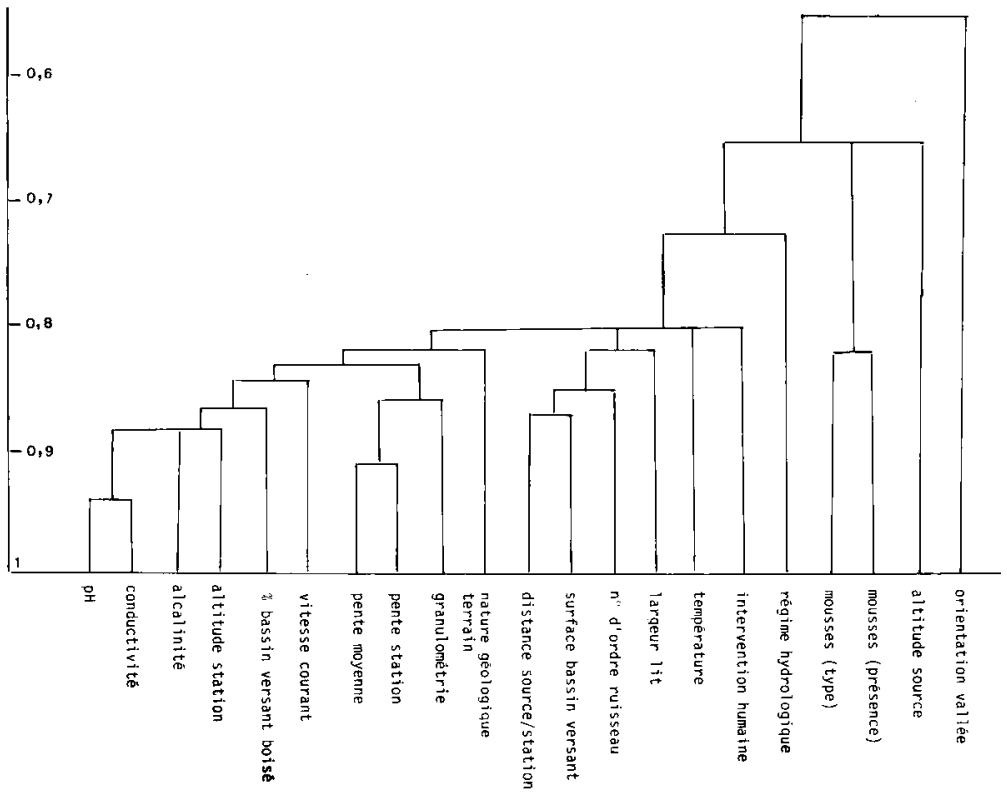

Fig. 6. Hiérarchisation des paramètres sur les cinq premiers axes.

Une première analyse a porté sur l'ensemble des 108 stations et sur le seul critère présence-absence des espèces. Les profils de chaque station s'écartent peu du profil moyen ; en effet, crues et dérive ont pour conséquence une redistribution permanente des organismes. Ces phénomènes se traduisent, dans chaque station, par un certain nombre d'espèces représentées seulement par 1 ou 2 individus, sans relations véritables avec les facteurs écologiques. Dans une seconde série d'analyses, nous avons éli. miné les espèces les plus rares (présentes dans 2 stations au plus) et les stations les plus pauvres ( 2 espèces au plus). Nous avons également considéré non plus le seul critère présence-absence, mais aussi l'abondance relative des espèces dans chaque station, suivant 6 classes de pourcentage :
- classe 1 : moins de $2 \%$ des individus

- classe $2: 2$ à $7 \%$

- classe $3: 7$ à $15 \%$

- classe $4: 15$ à $30 \%$

- classe $5: 30$ à $60 \%$

- classe 6 : plus de $60 \%$

Le nuage des points stations-espèces a la même forme que le nuage stations-paramètres de l'analyse précédente, et la position des stations par rapport aux axes est identique, à deux exceptions près (sur lesquelles nous reviendrons). Cette similitude nous a permis, dans une nouvelle série d'analyses factorielles, de traiter simultanément stations, espèces, paramètres et indices biospécifiques. 

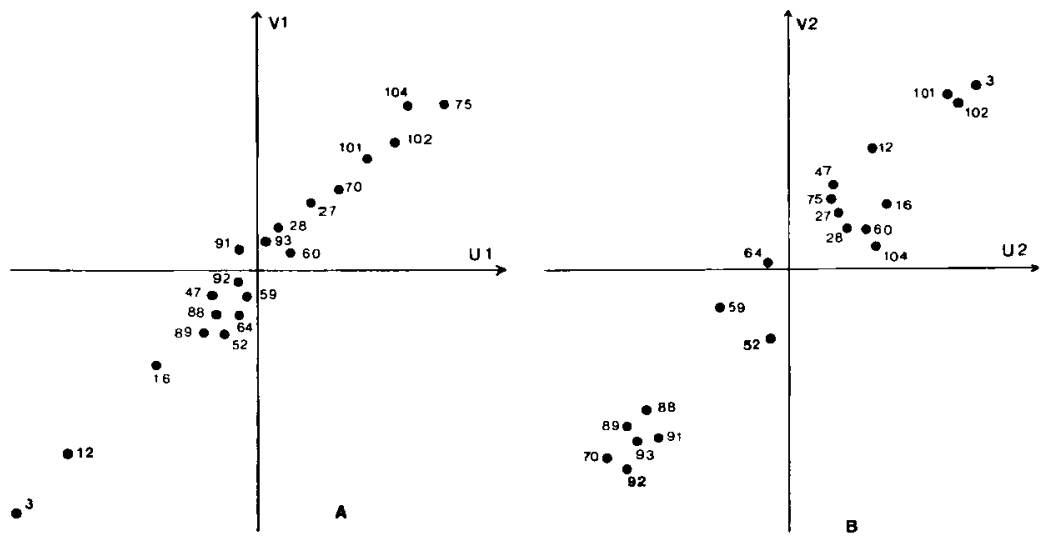

Fig. 7. Analyse canonique sur deux ensembles de paramètres écologiques et topographiques. $7 \mathrm{~A}$ : facteurs $U_{1}$ et $V_{1}, 7 \mathrm{~B}$ : facteurs $U_{2}$ et $V_{2}$.

3.1. - Analyse factorielle des correspondances stations-especes avec critères d'abondance (fig. 8 et 9)

Les 3 premiers axes rendent compte de $25,5 \%$ de l'inertie totale du nuage des points. Les espèces qui contribuent le plus à l'axe I (espèces structurantes) sont, par ordre décroissant :

a) positives sur l'axe:

Atractides gibberipalpis

Lebertia zschokkei

Feltria minuta

Sperchon denticulatus

Stations de la haute vallée d'Aure : $3,10,11,12,20$, 21, 23.

b) négatives sur l'axe :

Torrenticola amplexa

Torrenticola stadleri

Torrenticola madritensis

Torrenticola anomala

Lebertia fimbriata

Lebertia porosa

Torrenticola similis

Stations $93,94,95,91,78$ et 68 , c'est-à-dire un ensemble de stations des coteaux de Gascogne et de la basse vallée de la Neste.
Les espèces qui contribuent le plus à l'axe II sont :

a) positives sur l'axe :

Alurus scaber

Atractides nodipalpis

Aturus elongatus processiger

Sperchon hispidus

Stations $71,72,102,70,54$, sur la basse Neste d'Aure, le ruisseau de la Mousquère et le haut-Adour.

b) négatives sur l'axe:

Lebertia zschokkei

Torrenticola amplexa

Torrenticola stadleri

Lebertia dubia

Feltria minuta

Stations $94,93,11,10$ coteaux de Gascogne et sources d'altitude.

Les espèces qui contribuent le plus à l'axe III sont :

a) positives sur l'axe :

Aturus elongatus processiger

Panisus michaeli

Hygrobates calliger

Aturus scaber

Lebertia dubia

Kongsbergia materna

Stations $102,99,100,54,71$, c'est-à-dire des substrats de mousses. 

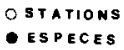

(1) Hte VALLEE D'AURE

(2.) MOYENNE VALLEE d'AURE

(3) BASSE VALLEE D'AURE

(a) Ht ADOUR

(5) $R$ des BARONNIES

(6) $R$ des cotreaux de Gascugane

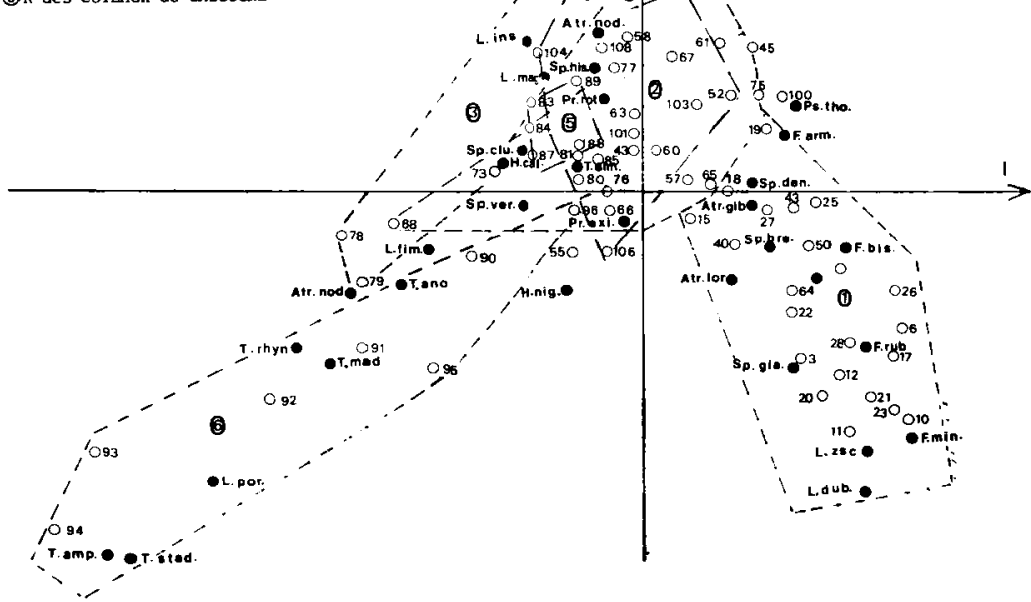

Fig. 8. Analyse factorielle des correspondances stations-especes avec critères d'abondance. Axes I et II.

b) négatives sur l'axe:

Sperchon hispidus

Sperchon denticulatus

Lebertia maglioi

Lebertia insignis

Stations $108,73,70,59$, c'est-à-dire des substrats de galets.

Sur l'axe I s'opposent stations et espèces de haute altitude aux stations et espèces des coteaux de Gascogne. Sur l'axe II s'opposent des espèces très spécialisées (qu'elles soient caractéristiques des sources de haute altitude ou des rivières de Gascogne) à des espèces à valence écologique plus large. Ces dernjères colonisent en effet aussi bien des eaux froides comme celles de la Mousquère ou du Camoudiet que des eaux plus chaudes comme celles de la Neste
d'Aure à Bizous. Enfin, sur l'axe III s'opposent les espèces muscicoles et pétricoles.

De façon globale, on peut distinguer trois zones de peuplement, de l'amont vers l'aval, avec pour dominantes respectives les espèces des genres $\mathrm{Fel}$. tria, Sperchon et Torrenticola. Les peuplements à dominantes Sperchon et Torrenticola ont déjà été signalés (Angelier 1953, 1957).

Suivant leur position sur l'axe I de l'analyse factorielle, on peut établir les successions amont-aval suivantes, pour quelques genres importants:

- Lebertia dubia $\rightarrow$ L. zschokkei $\rightarrow$ L.insignis

$\rightarrow$ L. maglioi $\rightarrow$ L. fimbriata

- Sperchon glandulosus $\rightarrow$ Sp. brevirostris $\rightarrow$ $S p$. hispidus $\rightarrow S p$. clupeifer. 


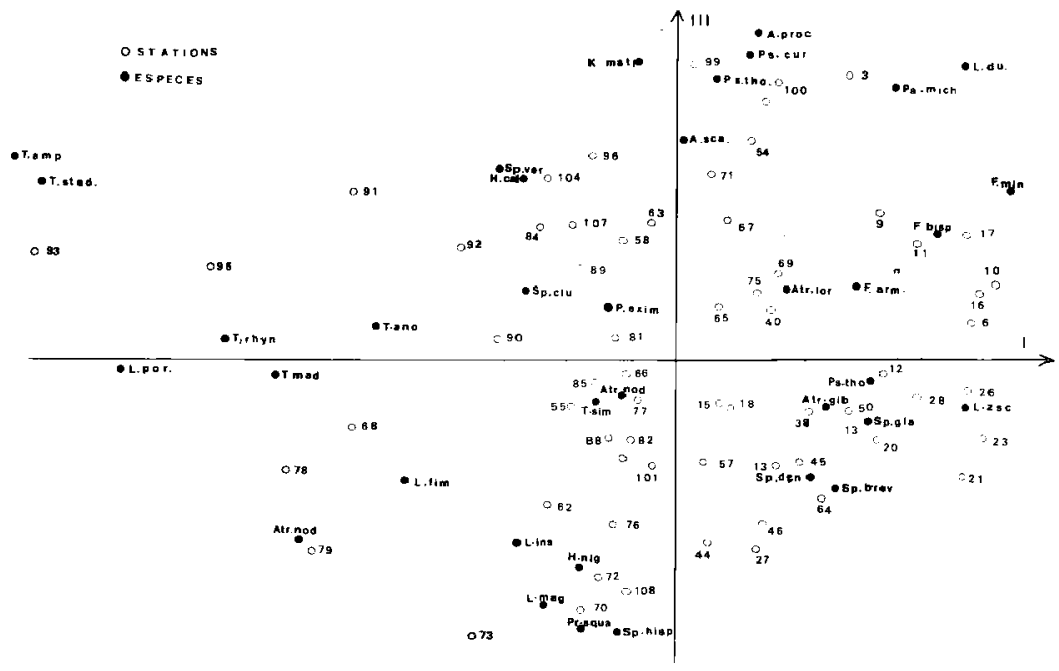

Fig. 9. Analyse factorielle des correspondances stations-espèces avec critères d'abondance. Axes I et III.

- Atractides loricatus $\longrightarrow$ A. gibberipalpis $\longrightarrow$

A. nodipalpis $\rightarrow$ A. nodipalpis pennatus

- Feltria minuta $\rightarrow$ F. rubra $\rightarrow$ F. bispinosa $\rightarrow F$. armata $\rightarrow F$. rouxi

- Torrenticola fagei $\rightarrow T$. similis $\rightarrow T$. madritensis $\longrightarrow T$. anomala $\longrightarrow T$. amplexa $\longrightarrow$ $T$. stadleri $\rightarrow T$. remyi

\section{2. - Analyse factorielle des correspondances stations-espèces-paramètres (fig. 10 à 12)}

Les 5 premiers axes rendent compte de $24,8 \%$ de l'inertie du nuage de points. Aux espèces structurantes de l'axe I (3.1.) correspondent :

- d'une part, positifs sur l'axe, l'altitude supérieure à $1100 \mathrm{~m}\left(\mathrm{~B}_{1}\right.$ et $\left.\mathrm{B}_{3}\right)$ et un environnement de pacages (étage subalpin, $S_{1}$ ), un régime des eaux nival $\left(R_{1}\right)$, nival de transition $\left(\mathbb{R}_{2}\right)$ ou de source $\left(\mathbf{R}_{5}\right)$.

- d'autre part, négatifs sur l'axe, des températures estivales supérieures à $15^{\circ} \mathrm{C}\left(\mathrm{O}_{5}\right)$, une pente inférieure à $5 \%\left(\mathrm{D}_{5}\right)$, un substrat de galets, un régime des eaux pluviothermique $\left(\mathrm{R}_{4}\right)$, des terrains mollassiques $\left(\mathrm{P}_{5}\right)$. Ce sont les caractères des rivières gasconnes.

On observe sur l'axe I les variations de la vitesse du courant telles qu'elles apparaissent le long d'une rivière à son profil d'équilibre : courants très lents $\left(E_{5}\right)$ correspondant aux sources limnocrènes ou au contraire très rapides $\left(E_{1}\right)$ aux altitudes les plus élevées, positifs sur l'axe. Vitesses moyennes $\left(\mathrm{E}_{3}, 25\right.$ à $50 \mathrm{~cm} / \mathrm{sec}$ ) vers l'origine de l'axe et vitesses lentes $\left(\mathrm{E}_{4}\right)$ négatives sur l'axe. Quant aux mousses, elles sont d'abord peu abondantes avec les Bryacées 


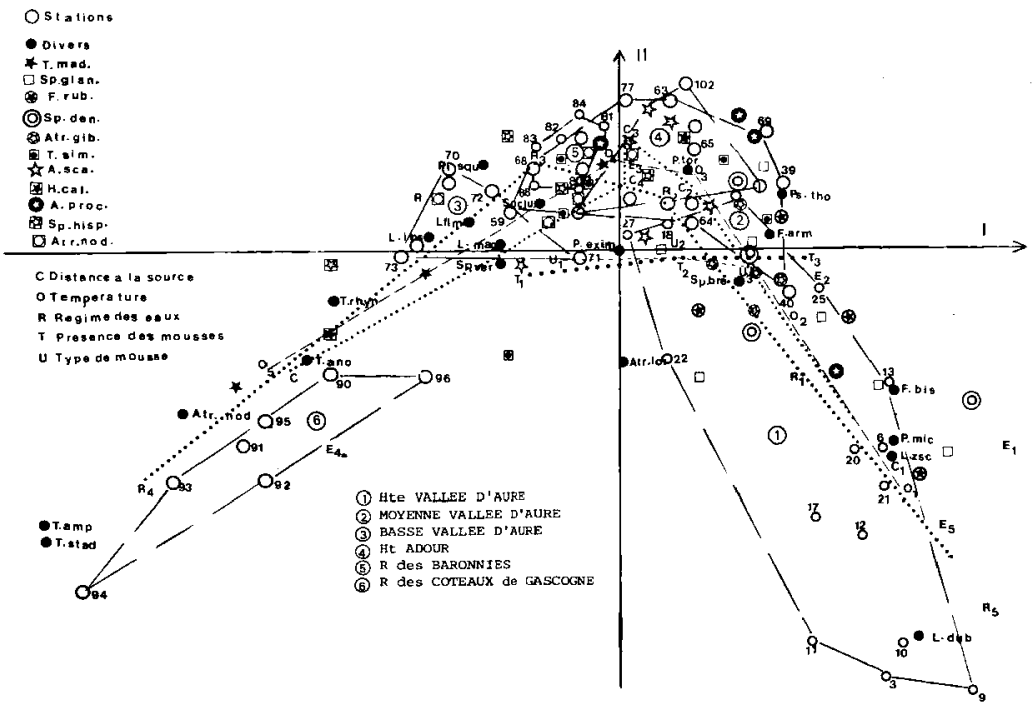

Fig. 10. Analyse factorielle des correspondances stations-espèces-paramètres. Axes I et II.

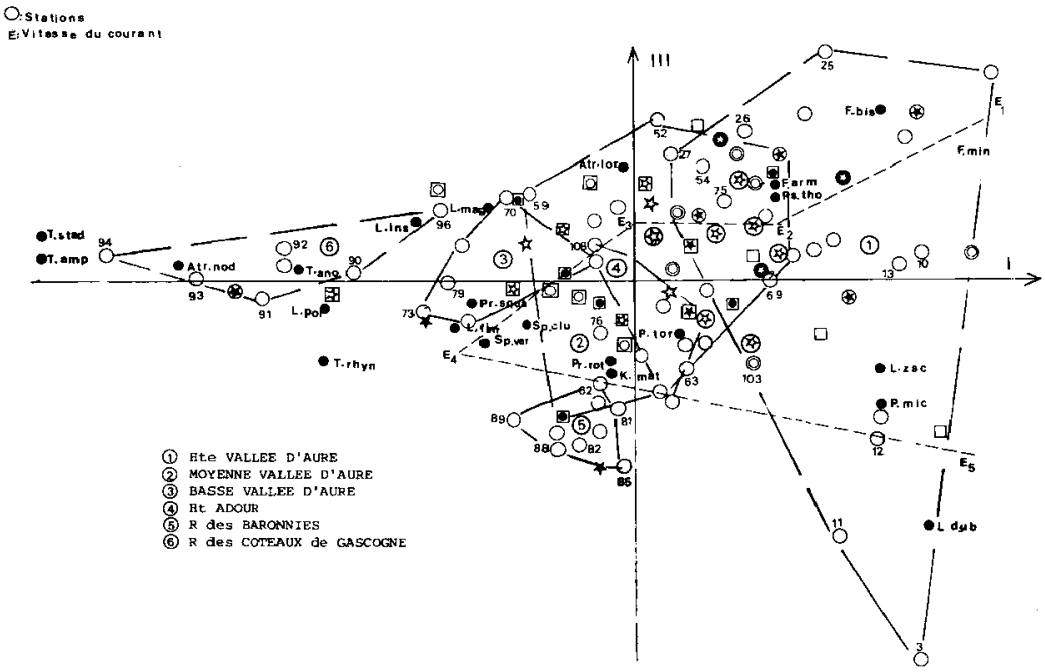

Fig. 11. Analyse factorielle des correspondances stations-espèces-paramètres. Axes I et II (Légendes. cf. fig. 1O) 


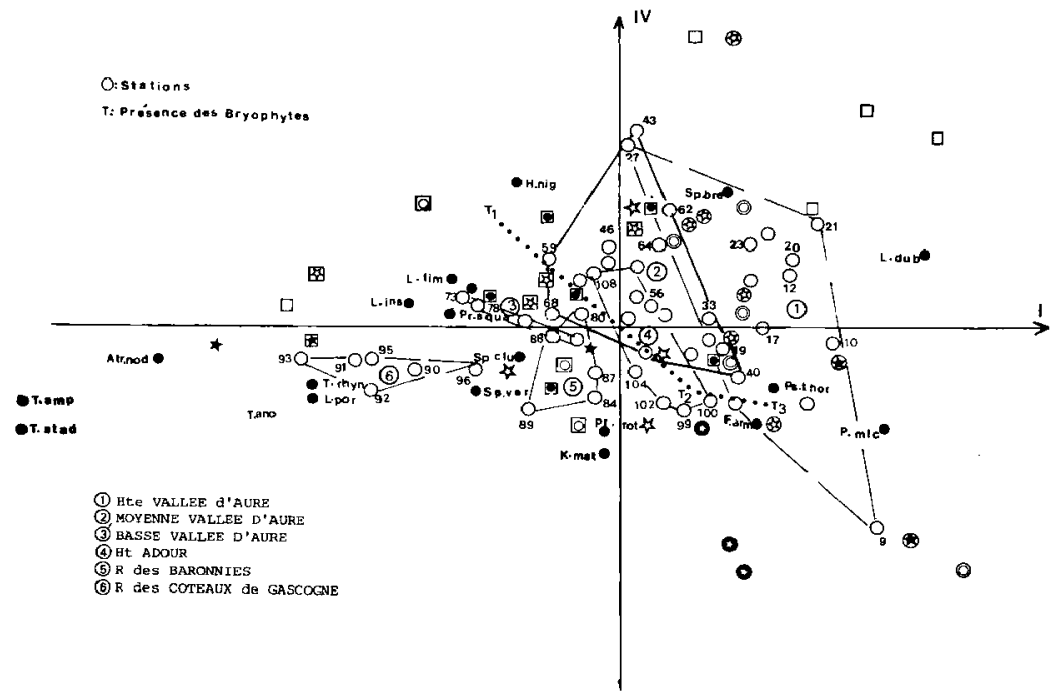

Fig. 12. Analyse factorielle des correspondances stations-espèces-paramètres. Axes I et IV (Légendes: cf. fig. 10)

dominantes $\left(\mathrm{U}_{2}\right.$ et $\left.\mathrm{T}_{2}\right)$; Hypnacées dominantes ou seules, occupant de larges surfaces, ensuite $\left(U_{3}\right.$ et $\mathrm{U}_{4}, \mathrm{~T}_{3}$.

Sur l'axe II s'opposent les espèces spécialisées de haute et basse altitude aux espèces eurytopes. Aux espèces spécialisées correspondent, en position négative sur l'axe:

- soit températures inférieures à $6^{\circ} \mathrm{C}\left(\mathrm{O}_{1}\right)$, distance source-station inférieure à $1 \mathrm{~km}\left(C_{1}\right)$, régime des eaux nival $\left(\mathbf{R}_{1}\right)$ ou de source $\left(\mathbf{R}_{5}\right)$, pour les espèces de haute altitude.

- soit températures supérieures à $15^{\circ} \mathrm{C}\left(\mathrm{O}_{5}\right)$, distance source-station supérieure à $40 \mathrm{~km}\left(\mathrm{C}_{5}\right)$, régime des eaux pluviothermique $\left(R_{4}\right)$.

Aux espèces eurytopes correspondent des températures estivales de 9 à $15^{\circ} \mathrm{C}\left(\mathrm{O}_{3}\right.$ et $\left.\mathrm{O}_{4}\right)$, un régime des eaux nival de transition $\left(\mathrm{R}_{2}\right)$ ou pluvio-nival $\left(\mathrm{R}_{3}\right)$.
L'inertie des axes III et IV est très proche $(4,19$ et $4,04 \%$ ). Sur l'axe III s'opposent essentiellement des espèces de haute altitude, - les unes de courant lent (négatives sur l'axe), les autres de courant rapide (positives sur l'axe). Les différentes classes de vitesse de courant suivent l'axe III (fig. 11), de $\mathrm{E}_{1}$ (très rapide, positif sur l'axe) à $E_{5}$ (très lent, négatif sur l'axe). Sur l'axe I, le paramètre vitesse du courant suivait l'axe, - simple paramètre amont-aval, - et les coordonnées des points représentant $E_{1}$ et $\mathbf{E}_{5}$ étaient proches : elles marquaient la proximité des sources limnocrènes et des cascades de haute altitude. Au contraire, sur l'axe III, ces coordonnées sont situées chacune à une ext rémité de l'axe. Espèces et stations de haute altitude sont largement diversifiées en fonction du courant, contrairement aux espèces et stations de basse altitude. L'axe III traduit finalement l'accroissement de la diversité des biotopes de haute altitude.

Quant à l'axe IV, il correspond en fait à l'axe III de l'analyse stations-espèces : il traduit l'opposition 
entre espèces muscicoles et pétricoles: Feltria et Aturus, muscicoles, en position négative sur l'axe et Sperchon positifs. Les espèces plutôt muscicoles à l'état nymphal et plutôt pétricoles à l'état adulte figurent en plusieurs points le long de l'axe IV (correspondant aux classes d'abondance). C'est notamment le cas de Sperchon denticulatus, Atractides gibberipalpis et Atractides nodipalpis.

\subsection{Associations d'Hydracariens et facteurs écologiques}

Les groupements de points représentant stations, espèces et parametres sur les différents axes permettent de reconnaître un certain nombre d'associations et de préciser leur déterminisme.

\section{Association I \\ Panisus michaeli \\ Sperchon glandulosus \\ Lebertia dubia \\ Lebertia zschokkei}

Stations-types : 3 et 11

\section{Association II}

\section{Lebertia zschokkei \\ Feltria minuta \\ Feltria rubra \\ Feltria bispinosa}

Stations types: 9 et 10

Ces deux associations caractérisent les ruisseaux de haute altitude. Elles sont corrélées, sur les axes I e1 II, aux températures inférieures à $6^{\circ} \mathrm{C}\left(\mathrm{O}_{1}\right)$, distance à la source inférieure à $1 \mathrm{~km}\left(C_{1}\right)$, et $B_{1}$ et $\mathbf{B}_{2}$. Elles se séparent dans les plans des axes I et III et I et IV. L'association II caractérise les mousses : genre Feltria et Hypnacées dominantes $\left(\mathrm{U}_{3}\right)$ sont liés, ainsi que le régime des eaux nival $\left(\mathrm{R}_{\mathrm{I}}\right)$. Lorsque le courant diminue, les Feltria régressent, tandis que Lebertia zschokket, puis L. dubia, Sperchon glandulosus et Panisus michaeli apparaissent successivement (association I), aussi bien en substrat pétricole que dans les mousses. Les indices de Fisher (faible) et de Simpson (élevé) rendent compte de la faible diversité et de la dominance d'un nombre réduit d'espèces.

\section{Association $\mathrm{III}$}

Panisopsis thori

Sperchon brevirostris

Sperchon denticulatus

Sperchon glandulosus

Atractides gibberipalpis

Atractides loricatus

Feltria armata

Stations-types : $15,19,25,27,33,39,40,43,45,48$, 50,61 .

Haute et moyenne vallée d'Aure, au-delà de $700 \mathrm{~m}$, haute vallée de l'Adour (mousses et galets).

L'association III est caractérisée par les espèces sténothermes du genre Sperchon. Elle est corrélée aux parametres altitude entre 800 et $1900 \mathrm{~m}$ (en fait, $1500 \mathrm{~m}, \mathrm{~B}_{2}$ et $\left.\mathrm{B}_{4}\right)$, températures entre 6 et $12^{\circ} \mathrm{C}\left(\mathrm{O}_{2}\right.$ et $\left.\mathrm{O}_{3}\right)$, pente inférieure à $30 \%\left(\mathrm{D}_{2}\right.$ et $\left.\mathrm{D}_{3}\right)$, régime des eaux nival ou nival de transition $\left(R_{1}\right.$ ou $R_{2}$ ), avec ou non la présence d'Hypnacées. A ce niveau, peuplements de mousses et de galets se confondent partiellement, - les Sperchon et Atractides gibberipalpis étant surtout muscicoles à l'état nymphal et pétricoles à l'état adulte. L'indice de Fisher est plus élevé que pour les associations I et II (de 2 à 5), tandis que l'indice de Simpson diminue $(0,3$ à 0,4$)$.

Association IV

Panisus torrenticolus

Panisopsis curvifrons

Aturus scaber

Aturus elongatus processiger

Kongsbergia materna

Stations-types : 54, 72, 75, 99, 102. Mousses de la basse et moyenne vallée d'Aure et du haut Adour.

C'est l'équivalent de l'association II, mais dans les mousses d'altitude plus basse. Le genre Aturus remplace le genre Feltria. Les températures estivales se situent entre 9 et $15^{\circ} \mathrm{C}\left(\mathrm{O}_{3}\right.$ et $\left.\mathrm{O}_{4}\right)$. Le régime des eaux est nival de transition ou pluvio-nival $\left(\mathbf{R}_{2}\right.$ ou $R_{3}$ ). Le courant ne semble pas jouer un rôle déterminant sur le peuplement.

Association $V$

Protzia squamosa

Sperchon hispidus

Lebertia insignis

Lebertia maglioi

Torrenticola similis

Atractides nodipalpis

Stations types: $59,70,73,108$. Galets de basse et moyenne altitude. 
Cette association est corrélée aux mêmes paramètres, altitude, température, régime des eaux, que l'association IV. Mais le substrat est constitué de galets, et non de mousses, et c'est sur l'axe III de l'analyse factorielle stations-especes que les deux associations se différencient nettement. Le courant joue un rôle important dans la distribution des espèces : Protzia squamosa et Lebertia insignis apparaissent lorsque la vitesse du courant diminue.

\section{Association VI}

Sperchon clupeifer

Sperchonopsis verrucosa

Torrenticola similis

Hygrobates calliger

Atractides nodipalpis

Stations-types : 80 à $89,104,107$. Ruisseaux des Baronnies et affluents de l'Adour prenant leur source vers $1200 \mathrm{~m}$. Substrats de mousses ou de galets.

Cette association se distingue essentiellement de la précédente sur l'axe III de l'analyse factorielle stations-espèces-paramètres. Elle est corrélée à une faible pente $\left(D_{5}, 5\right.$ à $\left.10 \%\right)$ et un courant lent $\left(E_{4}, 10\right.$ à $25 \mathrm{~cm} / \mathrm{sec}$ ), ainsi qu'à un régime des eaux pluvionival ou pluvial $\left(\mathrm{R}_{3}\right)$. A Torrenticola similis et Atractides nodipalpis de l'association V se joignent Sperchon clupeifer, Sperchonopsis verrucosa et Hygrobates calliger, qui peuvent tous être muscicoles au stade nymphal et pétricoles au stade adulte: les deux substrats sont donc confondus.

\section{Association VII}

\section{Torrenticola anomala}

\section{Torrenticola madritensis}

Sperchonopsis verrucosa

Lebertia fimbriata

Stations-type : $68,78,79,90,91,92,95$. Rivières des Coteaux de Gascogne, cours inférieur du Nistos, ruisseau de Beyrède (mousses et galets).

C'est l'association à Torrenticola anomala et $T$. madritensis, muscicoles (nymphes) ou pétricoles. Elle est liée aux paramètres altitude inférieure à $700 \mathrm{~m}\left(\mathrm{~B}_{5}\right)$, pente inféricure à $5 \%\left(\mathrm{D}_{6}\right)$ et régime des eaux pluvial ou pluviothermique $\left(\mathbf{R}_{3}\right.$ ou $\left.\mathbf{R}_{4}\right)$. L'indice de Fisher est supérieur à 6 et $l$ 'indice de Simpson inférieur à 0,1 .

\section{Association VIIJ}

\section{Lebertia porosa}

Torrenticola amplexa

Torrenticola stadleri

Hygrobates calliger

Atractides nodipalpis pennatus

Stations-types : 93 et 94 . Galets sur la Baïse et le Bouès.

Torrenticola amplexa et $T$. stadleri correspondent à un courant très faible et un régime des eaux pluviothermique. On est très près des associations des rivières méditerranéennes, aux étiages d'été prononcés. Par rapport à l'association précédente, il s'agit encore d'une réduction du courant : $T$, stadleri est une espèce fréquente dans les eaux stagnantes.

Les huit associations d'Hydracariens mises en évidence ne sont évidemment pas rigoureuses. Certaines espèces figurent dans plusieurs associations, par suite de leur large valence écologique, - Hygrobates calliger, par exemple.

De plus, ces associations reflétent mal l'opposition apparue sur l'axe III de l'analyse factorielle stations-espèces et l'axe IV de l'analyse stationsespèces-paramètres, entre Hydracariens pétricoles et muscicoles. Certaines associations caractérisent mousses ou galets seulement, tandis que d'autres caractérisent les deux substrats simultanément. Nous avons donc été amenés à réaliser une nouvelle série d'analyses, traitant séparément les stations en fonction de leur substrat.

\section{4. - Analyse factorielle des correspondances stations-espèces-paramètres des biotopes à substrats de galets}

L'analyse met en évidence 6 associations. Deux d'entre elles correspondent respectivement aux associations V et VIII de l'analyse globale (galets de basse et moyenne altitude et rivières gasconnes à courant lent). Les autres correspondent en partie aux associations I, III, VI et VII.

La signification des trois premiers axes n'est pas modifiée par rapport à l'analyse précédente (3.2). Les faits importants sont l'absence de faune pétricole pour des températures inférieures à $6^{\circ} \mathrm{C}\left(\mathrm{O}_{1}\right)$ et des courants supérieurs à $1 \mathrm{~m} / \mathrm{sec}\left(\mathrm{E}_{1}\right)$. Les espèces s'étirent le long de l'axe I. Seules se détachent 
sur l'axe II les quelques espèces des sources limnocrènes. Sur l'axe III s'individualise un petit nombre d'especes subsistant dans des courants de 0,5 à 1 $\mathrm{m} / \mathrm{sec}\left(\mathrm{E}_{2}\right)$ comme Sperchon denticulatus, Atractides gibberipalpis et $A$. loricatus.

Il existe un hiatus entre la zone à Lebertia dubia et $L$. zschokkei et celle a Sperchon denticularus et Atractides gibberipalpis : il correspond au brusque relèvement de la pente au niveau de l'étage montagnard dans les vallées glaciaires des Pyrénées centrales. La distribution des Hydracariens pétricoles est très liée au courant, et par conséquent à la pente.

Le régime des eaux esı également un paramètre structurant l'axe III.

\section{5. - Analyse factorielle des correspondances stations-espèces-paramètres des biotopes à substrats de mousses}

L'analyse met en évidence 6 associations. Trois d'entre elles correspondent respectivement aux associations I, II el IV de l'analyse globale. Les autres correspondent en partie aux associations III, VI et VII. Les Hydracariens spécifiques des mousses (Feltria, Aturus, Kongsbergia,...) colonisent des eaux plus fraîches que les Hydracariens pétricoles : les associations muscicoles dominent sur les cours supérieurs et moyens des cours d'eau. Sperchon denticlatus et Atractides gibberipalpis n'apparaissent qu'à partir de l'association III. L'opposition mousses-galets s'estompe dans les basses vallées (associations VI et VII).

La signification des deux premiers axes est la même que dans l'analyse globale. Sur l'axe III s'opposent des stations muscicoles du bassin de la Neste d'Aure (jusqu'à Lortet), aux stations des Baronnies (négatives sur l'axe). L'altitude de la source $\left(\mathrm{A}_{2}\right.$, audelà de $2200 \mathrm{~m}$ ou $A_{4}$, au-dessous de $1900 \mathrm{~m}$ ), le régime des eaux ( $R_{1}$, nival, ou $R_{3}$, pluvio-nival), le numéro d'ordre du ruisseau et la surface du bassin versant sont les paramètres structurant l'axe III.

Sur l'axe IV s'opposent seulement Lebertia dubia et Panisus michaeli à Lebertia zschokkei et Feltria minuta c'est-à-dire les espèces de sources limnocrènes ou de courant de haute altitude.

\section{6. - Conclusions}

L'association I caractérise les sources limnocrènes, pratiquement sans courant. Elle apparait dans l'analyse factorielle globale, isolée sur les axes III et IV, et dans l'analyse sur substrats de mousses. Sur les 7 autres associations, 2 sont strictement muscicoles (II et IV), 2 sont strictement pétricoles (V et VIII) et 3 sont mixtes (III, VI et VII). L'opposition Hydracariens muscicoles et pétricoles, nette sur le cours supérieur des cours d'eau, s'estompe à basse altitude.

Le rôle du courant est fondamental dans la répar. tition des espèces pétricoles. Mais celles-ci vivent habituellement sous les pierres, et un certain nombre s'enfoncent dans le sous-écoulement. Courant et pente sont étroitement corrélés, ainsi que l'a montré l'analyse canonique (2.4). Il semble que leur action se traduise, indirectement, par une instabilité du substrat (notamment lors des crues).

Il en est tout autrement des mousses. Elles se développent sur des substrats stables : roche-mère, gros blocs de rochers du cours supérieur des coursd'eau, - mais aussi, à toutes altitudes, sur des rives consolidées par l'homme, piles de ponts, petites retenues. Dans la hiérarchisation des paramètres tirée de l'analyse factorielle (2.3.), les mousses apparaissent relativement indépendantes. C'est peut-être parce-que aucun des paramètres pris en compte ne caractérise la pérennité, qui lie entre eux des substrats comme la roche-mère, les gros blocs de rochers, les rives consolidées, les piles de ponts....

Dawson (1973) a montré, dans une station située près de la Neste de Couplan, que la majorité des plants de Fontinalis squamosa étaient âgés de 3 à 5 ans, - quelques-uns atteignant 9 ans. Ceci souligne la nécessité d'un substrat stable dans le temps pour le développement des mousses.

L'influence du substrat de mousses sur le peuplement en Hydracariens se manifeste de deux façons. Lorsque les Hypnacées sont seules ou dominantes, en courant rapide, les Feltria ou Aturus sont abondants. Le peuplement des Bryacées (notamment des Fissidens) est pratiquement nul lorsqu'elles sont seules ou dominantes. Les rameaux des Bryacées sont à peu près parallèles à leur tige, qui est dressée ; les feuilles sont en forme de lame (Fissidens) ou d'aiguille (Polvtrichum). Les Hypnacées sont au contraire caractérisées par des rameaux non parallèles aux 
tiges ; celles-ci sont souvent couchées sur le substrat. Les feuilles ont la forme de cupules imbriquées les unes dans les autres (en particulier dans le genre Hypnum). Les Hypnacées offrent aux Hydracariens strictement muscicoles, - qui sont de très petite taille, - une bonne protection contre le courant, contrairement aux Bryacées. Elles assurent également, par leur mode de vie. la continuité dans le temps du biotope, contrairement aux substrats de galets.

Il est possible d'expliquer ainsi le peuplement des eaux courantes par les différentes associations d'Hydracariens :

- Dans les sources limnocrènes de haute altitude, le peuplement est constitué par des formes qui sont pratiquement d'eaux stagnantes, vivant indifféremment sur substrats de mousses ou de galets (association I).

- Sur le cours supérieur des rivières, à forte pente, le peuplement des mousses est assuré par les genres Feltria (association II) ou Aturus-Kongsbergia (association IV) suivant l'altitude.

- Lorsque la pente est faible, substrats de mousses et de galets sont situés côte à côte, et les nymphes des especes pétricoles colonisent les mousses, éventuellement les adultes si le courant est très lent, - de sorte que l'opposition mousses-galets s'estompe. Le courant joue un rôle important dans la répartition des espèces pétricoles : celles-ci sont éliminées des mousses lorsque sa vitesse s'accroît. Mais il ne paraît pas jouer un rôle important dans la répartition des espèces strictement muscicoles, - au moins lorsqu'elles colonisent les Hypnacées.

Gazagnes (1983) et Bouguenec \& al. (1984) ont étudié la dérive des Invertébrés sur la Neste d'Aure et ont recueilli 48 espèces d'Hydracariens et Halacariens (dont 4, représentées chacune par un individu, ne figuraient pas dans nos stations).

Dans la haute vallée de la Neste, 3 genres (11 espèces sur 28), - Sperchon, Atractides et Torrenticola, - représentent $85,3 \%$ de la dérive. 4 espèces sur 11. - Sperchon denticulatus, Atractides gibberipalpis, Torrenticola similis et Atractides nodipalpis constituent à elles seules $76,5 \%$ de la dérive.

Dans la basse vallée de la Neste, en octobre, les genres Sperchon (37\%) et Lebertia (12,2\%), Torren. ticola similis (10\%) Atractides gibberipalpis et $A$. nodipalpis $(24,6 \%)$ et Aturus scaber $(9,7 \%)$ représentent $93,5 \%$ des Hydracariens de la derive.
Les espèces dominantes de la dérive ont presque toutes un caractère commun : ce sont des formes pétricoles colonisant la partie superficielle du subs. trat, instable (seul Torrenticola colonise également la zone profonde). Les deux espèces les plus abondantes sur la haute Neste d'Aure sont les seules qui subsistent lorsque la vitesse du courant dépasse $1 \mathrm{~m} / \mathrm{sec}$. Les espèces muscicoles (à l'exception de Aturus scaber dans la basse vallée de la Neste) ne dérivent qu'accidentellement.

\section{4. - La réalité des facteurs écologiques}

Réunir dans une même analyse factorielle les espèces, les stations et un ensemble de paramètres physico-chimiques, topographiques ou autres, ris. que de donner une image déformée de la réalité, du rổe des différents facteurs écologiques dans la répartition des Hydracariens. Et celà, même si les coordonnées des stations sur les différents axes sont proches dans les analyses séparées stations-espèces et stations-paramètres. Or, dans deux cas, - les Baronnies et la basse vallée d'Aure, - les coordonnées des stations sur les axes diffèrent suivant qu'elles ont été déterminées par les paramètres ou les espèces seulement. D'autre part, la hiérarchisation des paramètres sur 2 et 5 axes de l'analyse factorielle des correspondances a montré un certain nombre de corrélations entre ceux-ci, - notamment entre ceux qui varient régulièrement de l'amont vers l'aval. Une analyse canonique sur deux ensembles de paramètres quantifiables, - topographiques et écologiques, - a mis en évidence leur liaison : pente et vitesse du courant d'une part, altitudes de la source et de la station, surface du bassin versant et température d'autre part.

Nous avons, dans une série d'analyses factorielles, éliminé certains paramètres, afin de tenter de comprendre les problèmes posés par les Baronnies et la basse vallée d'Aure, et aussi de vérifier si un nombre de paramètres réduit, constituant un complexe stationnel, suffit à expliquer la répartition des Hydracariens.

\section{1. - Coordonnées des stations de la basse vallée de la Neste et des Baronnies sur les axes I, II et III}

Dans l'analyse factorielle stations-espèces (fig. 8 et 9), qui reflète la réalité écologique, le nuage de points représentant les stations sur les axes I et II 
a la forme d'une parabole dont le sommet est situé à l'extrémité positive de l'axe 11 . Les stations 70 et 72 (Neste à Lortet) sont situées au sommet de la parabole et, sur la branche gauche de celle-ci (négative sur l'axe I), se succèdent les stations du haut Adour (102 à 108), des Baronnies (80 à 89 ) et de la Neste à Bizous (st. 73), positives sur l'axe II ; puis les ruisseaux de piémont affluents de la Neste à basse altitude : ruisseau de Beyrède (st. 68) et bas Nistos (78 et 79), négatifs sur l'axe II. La station de Bizous (73), sur la Neste d'Aure, est nettement séparée de celle de Lortet, cependant très proche, et se rapproche du bas Nistos.

Dans l'analyse stations-paramètres (fig. 2 à 4), la succession sur la branche gauche de la parabole (axe II) devient : stations des Baronnies - bas Nistos et Neste d'Aure à Grézian (st. 59) - Lortet et Bizous (st. 70 à 73) et Coteaux de Gascogne. Les paramètres distance source-station (C) et surface du bassin versant (I) ont un poids trop important dans l'analyse et permettent difficilement de comparer des cours d'eau de longueur très différente. Ils rapprochent, par leur valeur absolue, le bas Nistos et la Neste d'Aure à Grézian, Lortet et Bizous aux cours d'eau des Coteaux de Gascogne.

L'analyse stations-espèces-paramètres (fig. 10 à 12) rétablit la parenté faunistique entre la Neste à Lortet et Grézian et le haut Adour. Mais il reste à expliquer la parenté faunistique entre la Neste à Bizous, le ruisseau de Beyrède et le bas Nistos (st. 73, 68, 78 et 79 ).

Au niveau de Sarrancolin (en amont de Lortet), un canal détourne une partie importante des eaux de la Neste d'Aure vers les Coteaux de Gascogne (soutien du débit des rivières gasconnes et irrigation). Le débit estival de la Neste d'Aure, en aval de Sarrancolin, ne correspond plus au débit naturel.

Ainsi, en juillet 1982, le débit moyen de la Neste à Sarrancolin était de $21 \mathrm{~m}^{3 / \mathrm{sec}}$, celui du canal de dérivation de $13,6 \mathrm{~m}^{3 /} / \mathrm{sec} ; 7,4 \mathrm{~m}^{3 /} \mathrm{sec}$ seulement coulaient dans la Neste à Lortet et Bizous. En août, les débits moyens étaient respectivement de $16,5 \mathrm{~m}^{3 /} / \mathrm{sec}$, $8,3 \mathrm{~m}^{3} / \mathrm{sec}$ et $6,8 \mathrm{~m}^{3} / \mathrm{sec}$.

Les stations de Lortet (70 à 72) sont proches de Sarrancolin, et la vallée de la Neste encaissée : les eaux se réchauffent peu, et la faune est encore celle de la moyenne vallée (proche de celle de Grézian, st. 59). Par contre, en aval de Lortet, le lit de la Neste s'élargit, la vitesse du courant diminue et les eaux se réchauffent. Les paramètres topographiques et hydrologiques utilisés dans l'analyse factorielle ne traduisent pas la modification des facteurs écologiques : les conditions du milieu sur la Neste à Bizous s'apparentent à celles du bas $\mathrm{Nistos}_{\text {, }}$ et le peuplement en Hydracariens reflète cette parenté.

La comparaison, sur une rivière, entre le peuplement théorique correspondant à urı certain nombre de paramètres topographiques et écologiques et le peuplement observé pourrait être un bon indicateur de l'impact humain (aménagements et pollutions) sur les eaux.

Les ruisseaux des Baronnies (st. 80 à 89 ) sont isolés dans les analyses stations-paramètres, particulièrement sur l'axe III (qui traduit à la fois le régime des eaux et les composantes chimiques). Cet isolement est démenti par l'analyse stations-espèces. Si nous supprimons, dans l'analyse stations. paramètres, la nature géologique du terrain $(\mathrm{P})$ et les paramètres qui en découlent $(\mathrm{pH}$, conductivité et alcalinité), l'isolement des stations des Baronnies disparât. Les facteurs chimiques ne semblent pas influencer la distribution des Hydracariens (au moins dans les massifs étudiés).

\section{2. - Définition d'un complexe stationnel par un nombre réduit de paramètres.}

Nous avons tenté de définir un complexe stationnel, stable pour une station et susceptible d'être corrélé aux facteurs écologiques, à l'aide d'un minimum de paramètres topographiques et hydrologiques. Pour simplifier les analyses factorielles, très nombreuses, qui ont du être effectuées, nous n'avons pris en compte que les paramètres et stations, et comparé les coordonnées des stations sur les axes à celles de l'analyse stations-espèces.

Une première analyse a été effectuée avec quatre paramètres seulement, qui paraissent représenter la plupart des autres (2.3) : altitude (B) et pente (H) de la station, surface du bassin versant (I) et régime des eaux (R). Les stations de Lortet et Bizous, sur la Neste d'Aure, se rapprochent alors de celles des Coteaux de Gascogne. Si nous remplaçons le régime des eaux par l'altitude de la source (A), la distribution des points représentant les stations sur les axes I et II n'a plus aucun rapport avec celle de l'analyse stations-espèces : les stations de la Neste de Badet et du haut Adour sont confondues ; Lortel et Bizous 


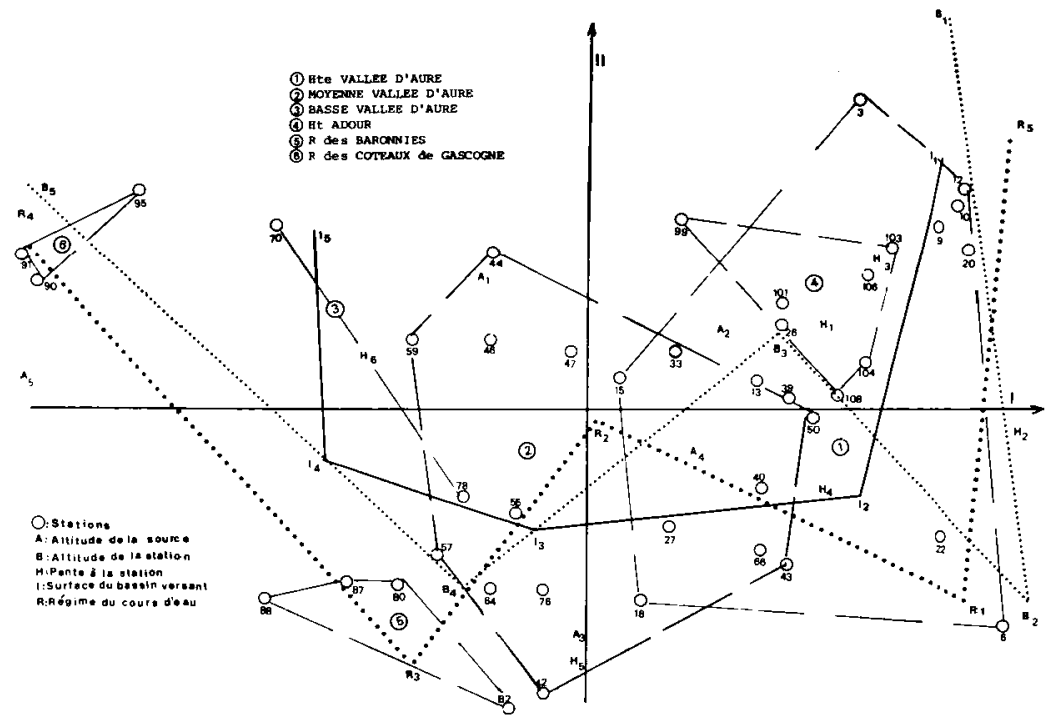

Fig. 13. Analyse factorielle des correspondances stations et paramètres altitude de la source (A), altitude de la station (B), pente de la station $(\mathrm{H})$, surface du bassin versant (I) et régime des eaux (R). Axes I et $I$.

sont situés au mêrne niveau. Le régime des eaux apparaît comme l'un des facteurs fondamentaux de la distribution des Hydracariens (régimes nival et nival de transition sont d'ailleurs confondus). Les pluies étant plus abondantes en hiver, cette saison est celle des crues lorsque l'écoulement de l'eau est immédiat. Les crues sont au contraire de printemps et début d'été lorsque les précipitations tombent sous forme de neige et que leur écoulement est différé.

Nous avons remplacé le régime des eaux, dans une nouvelle série d'analyses, par la durée moyenne de l'enneigement (de 5 mois au moins au niveau des stations de ski de la vallée d'Aure et au-dessus jusqu'à l'enneigement nul des Coteaux de Gascogne). $\mathrm{Ce}$ paramètre est homologue du régime des eaux. Dans une analyse comportant régime des eaux et durée de l'enneigement, le poids des deux facteurs est trop important : les stations des Coteaux de Gascogne et des Baronnies s'opposent à l'ensemble haute et moyenne vallée d'Aure et haut Adour, dont les stations sont plus ou moins confondues.

L'analyse stations-paramètres qui se rapproche le plus de la référence stations-espèces comporte cinq paramètres (fig. 13) :

- l'altitude de la source (A)

- l'altitude de la station (B)

- la pente de la station $(\mathbf{H})$

- la surface du bassin versant (I)

- le régime des eaux (R)

Un seul paramètre doit rendre compte de la position de la station sur le profil de la rivière (bassin versant, distance source-station ou bien numéro d'ordre du cours d'eau) pour que le poids de celuici ne soit pas trop important.

La parabole constituée par le nuage des points représentant les stations est la même que dans l'analyse stations-espèces, mais son sommet est inversé par rapport à l'axe Il. Les relations mises 
en évidence dans l'analyse canonique (2.3) entre une série de paramètres topographiques et certains facteurs écologiques sont confirmées (outre la nécessité de faire intervenir le régime des eaux).

Les cinq paramètres retenus sont tirés des deux premiers axes de l'analyse factorielle des correspondances. Ils ne séparent évidemment pas les espèces muscicoles et pétricoles, puisqu'ils ne font pas inter. venir le substrat.

\section{3. - Paramètres topographiques et hydrologiques et règle des pentes de Huet}

La règle pente-largeur de Huet $(1946,1949)$ permet de définir la zone à truites (Salmo fario) sur le profil en long d'un cours par des paramètres correspondant à peu près à l'axe I de nos analyses. Mais Huet ne tient pas compte du régime des eaux et de l'altitude de la station (qui peuvent rendre compte de la température à l'étiage). - alors que nous n'avons pu les éliminer de nos propres analyses.

Peut-être la règle pente-largeur résulte-t-elle d'études menées dans une même région, sur des cours d'eau au régime identique. Le fait que cette règle ne puisse s'appliquer aux rivières de Normandie (Cuinat 1971), aux eaux froides toute l'année, vient à l'appui de cette hypothèse. $D$ 'autre part, la règle pentelargeur rend compte de la distribution d'une seule espèce, Salmo fario, alors que les Hydracariens présentent des types biologiques très divers.

\section{4. - Diptères Athericidae et Rhagionidae et facteurs écologiques}

Lauga \& Thomas (1978) ont étudié l'écologie des larves de Diptères Athericidae et Rhagionidae cans le sud de la France. L'analyse factorielle des correspondances fait apparaître quatre facteurs de répartition des espèces:

- un facteur essentiel, de physionomie amont aval d'un cours d'eau, lié à la pente et à la température. Ce facteur correspond à l'axe I de nos analyses, et aussi à la règle des pentes de Huet. Mais l'altitude le définit moins pour les Athericidae et Rhagionidae que pour les Hydracariens. Celà tient à ce que ces Diptères, en altitude, sont cantonnés aux érnissaires des lacs, aux eaux réchauffées ; ils ne colonisent pas les sources et ruisseaux froids.

- un facteur (axe II) lié aux Bryophytes, qui semblent intervenir essentiellement comme sites de nymphoses, alors que les mousses correspondent à un substrat pour tous les stades de certains Hydracariens.

- un facteur (axe III) classant les espèces en fonction de leur « spécialisation".

- un facteur (axe IV) qui classe Athericidae et Rhagionidae en fonction de leur aptitude à survivre en courant rapide ou en eau stagnante.

Les deux groupes de Diptères étudiés ne comportent qu'un nombre réduit d'espèces et sont beaucoup moins diversifiés que les Hydracariens sur le plan écologique. La colonisation des eaux de haute altitude, notamment, est limitée à un cas très précis et on peut considérer Athericidae et Rhagionidae beaucoup plus comme des formes de piémont que comme des formes de massifs centraux.

\section{5. - Les types biologiques d'Hydracariens}

On réunit dans un même type biologique des espèces présentant des accomodements morphologiques et biologiques semblables, à des exigences d'habitat semblables.

\section{1. - Types biologiques et substrat}

5 grands genres peuplent l'horizon superficiel des galets : Protzia, Sperchon, Lebertia, Hygrobates et Atractides du groupe nodipalpis-gibberipalpis. Ils sont caractérisés par un corps globuleux, à tégument peu chitinisé, une taille relativement importante ( 800 à $2000 \mu$ suivant l'espèce et le sexe). Leur distribution est très inféodée à la pente.

Les espèces du genre Torrenticola sont également pétricoles, mais pénètrent dans le sous-écoulement et font donc partie du milieu hyporhéique. Le corps est aplati, le tégument très chitinisé, et la taille ne dépasse pas $900 \mu$.

Tous ces genres sont les éléments les plus constants de la dérive dans la vallée d'Aure.

Un certain nombre d'Hydracariens sont strictement inféodés au milieu hyporhéique. La plupart ne dépassent pas $400 \mu$, dans les familles des Feltriidae, Axonopsidae et Aturidae. Les Hydracariens muscicoles appartiennent à ces mêmes familles : leur taille réduite leur permet de trouver dans les mousses comme les Hypnacées une protection contre le courant. Pour la même raison, les espèces 
hyporhéiques peuvent coloniser les interstices du milieu hyporhéique.

Les types biologiques d'Hydracariens, en fonction du substrat, correspondent à peu près aux types biologiques des Invertébrés du sol:

Hydracariens muscicoles - faune muscicole Hydracariens pétricoles (horizon superficiel) faune de la litière

Hydracariens hyporhéiques facultatifs (type Torrenticola) - faune édaphique

Hydracariens hyporhéiques stricts - faune endogée.

\section{2. - Types biologiques et nombre d'œufs}

Le nombre d'cufs que renferme une femelle ne donne évidemment qu'une idée relative de son taux de reproduction. Il ne fait aucun doute que les Hydracariens présentent plusieurs pontes succes. sives, rapprochées, durant la période de reproduction, et que certaines espèces ont plusieurs généra. tions par an.

Il n'en demeure pas moins que les $\emptyset$ des espèces colonisant l'horizon supérieur des galets sont carac. térisées par un nombre d'cufs relativement élevé :

$\begin{array}{llll}\text { Lebertia porosa } & \text { jusqu'à } & 36 & \text { œufs } \\ \text { Lebertia dubia } & " & 25 & " \\ \text { Lebertia insignis } & " & 20 & \text { " } \\ \text { Atractides fluviatilis } & " & 20 & \text { " } \\ \text { Atractides nodipalpis } & " & 15 & \text { " } \\ \text { Protzia invalvaris } & " & 10 & \text { " } \\ \text { Atractides gibberipalpis } & \text { " } & 10\end{array}$

Par contre, dans les genres Torrenticola, Feltria, Axonopsis, Aturus et Kongsbergia, Atractides du groupe loricalus, le nombre d'oufs par ponte est réduit, -1 à 3 en général.

Deux types biologiques d'Hydracariens paraissent donc s'opposer par le nombre des aufs. Protzia, Sperchon, Lebertia, Hygrobates, Atractides du groupe nodipalpis-gibberipalpis peuvent être considérés comme des formes pionnières, colonisant l'horizon superficiel des fonds de galets, instable et souvent remanié.

Les espèces strictement muscicoles ou hyporhéjques, à nombre d'œufs réduit, colonisent des subs. trats dont la continuité dans le temps est assuré ; leurs pertes par dérive sont faibles. La continuité temporelle est assurée dans les mousses, car elles sont implantées sur des substrats stables ou stabilisés par l'homme ; elle est également assurée dans le milieu hyporhéique, car la migration en profondeur permet d'échapper à la dérive ou aux crues. Le genre Torrenticala présente le phénomène de dérive, mais sa survie est assurée par les individus du milieu hyporhéique, malgré son faible taux de reproduction.

\section{3. - Types biologiques et cycles biologiques}

La présence et l'abondance des nymphes, la présence et l'abondance des $९$ ovigères et l'évolution du sex-ratio (les $O$ adultes apparaissent plus précocement que les $Q$ et disparaissent plus tôt) nous renseignent sur le cycle biologique des Hydracariens.

La plupart des espèces présentent une génération annuelle: les ceufs éclosent au printemps, les nymphes apparaissent en juin-juillet et les $\$$ ovigères à la fin de l'été. Après la période de ponte, les adultes disparaissent progressivement. En altitude, le cycle est retardé. Le cycle peut comporter plusieurs générations dans l'année chez un certain nombre d'espèces, - ou bien il peut dépasser une année. Selon Bader (1968), le cycle de Sperchon glandulosus s'étend sur 3 ans dans une source à $600 \mathrm{~m}$ du Jura suisse.

Sur le tableau IV figurent les pourcentages des nymphes par rapport à la population totale, du printemps à l'hiver, toutes espèces confondues. En règle générale, les nymphes sont plus abondantes dans les mousses que sous les galets (sauf dans la haute vallée de l'Adour, en été). Les pourcentages de nymphes les plus élevés se situent:

- de l'automne au début du printemps dans la haute vallée d'Aure,

- à la fin du printemps et en été dans la vallée d'Aure, au-dessous de $1000 \mathrm{~m}$.

- au printemps dans les Baronnies

- en été sur les ruisseaux des Coteaux de Gascogne et la haute vallée de l'Adour.

Les données sont insuffisantes en hiver dans les Baronnies et le haut Adour, et au printemps sur les cours d'eau des Coteaux de Gascogne. Il apparaît néanmoins une relation étroite entre le déroulement du cycle des Hydracariens et la période d'étiage.

Sur les rivières des Baronnies, le cycle débute au printemps, dès la fin de la période des hautes eaux, - 


\begin{tabular}{|c|c|c|c|c|c|c|c|c|c|}
\hline & \multicolumn{3}{|c|}{ PRINTF:MPS } & \multicolumn{3}{|c|}{ ÉTÉ } & \multicolumn{3}{|c|}{ HIVER } \\
\hline & Galets & Mousses & $\begin{array}{l}\text { Galets et } \\
\text { mousses }\end{array}$ & Galets & Mousses & $\begin{array}{l}\text { Galets et } \\
\text { mousses }\end{array}$ & Gálets & Mousses & $\begin{array}{l}\text { Galets et } \\
\text { mousses }\end{array}$ \\
\hline $\begin{array}{l}\text { VALLEE D'AURE } \\
\text { (au-dessus de } 1000 \mathrm{~m} \text { ) }\end{array}$ & & & 36,6 & 17 & 22,5 & 20,3 & & & 29,7 \\
\hline $\begin{array}{l}\text { VALLEE D'AURE } \\
\text { (audessous de } 1000 \mathrm{~m} \text { ) }\end{array}$ & 14,6 & 29 & 21,8 & 16,8 & 25 & 22,3 & 9,4 & 10,8 & 8,8 \\
\hline HAUT-ADOUR & 0 & 5.4 & 2,1 & 22,7 & 3,5 & 17.5 & & & \\
\hline BARONNIES & 13,8 & 46 & 31 & 2,3 & 15,8 & 9,6 & & & \\
\hline COTEAUX DE GASOOONE & & & & 8,4 & 28,5 & 14,2 & 2,8 & 4 & 3 \\
\hline
\end{tabular}

Tableau IV. Pourcentage des nymphes par rapport à la population totale dans les bassins étudiés.

un peu plus tard dans la haute vallée de l'Adour. Dans la vallée d'Aure, au-dessous de $1000 \mathrm{~m}$, le cycle débute au printemps dans les ruisseaux de la basse vallée, en été sur la Neste (où il peut se poursuivre jusqu'en hiver). Dans la haute vallée d'Aure, le cycle débute en juillet-août, à la fin de la fonte des neiges, et il est d'autant plus tardif que l'altitude est plus élevée ; la proportion des nymphes est par conséquent élevée de la fin de l'été au début du printemps (avant le début de la fonte des neiges). Sur les rivières de Gascogne, l'abondance des nymphes en été est due essentiellement aux espèces du genre Torrenticola (sauf Torrenticola similis) ; elle traduit un peuplement proche de celui des rivières des Pyrénées orientales, aux étiages d'été accentués, caractérisé par le genre Torrenticola.

\subsection{1. - Cycle de Torrenticola similis}

Les nymphes apparaissent en avril sur les rivières des Baronnies, - à la fin de l'été seulement en moyenne vallée d'Aure. Sur le ruisseau de Morère (haut Adour), elles apparaissent de la fin du printemps au début de l'été. $T$. similis présenterait donc une génération annuelle, - le cycle étant retardé en altitude. Les adultes disparaissent progressivement de la fin de l'automne à l'hiver.

Protzia invalvaris, Sperchon clupeifer, Lebertia maglioi, Hygrobates calliger, Atractides nodipalpis, ont un cycle analogue, retardé en altitude.

Il n'est donc pas possible de conclure à l'existence de plusieurs générations annuelles chez. Hygrobates calliger et Atractides nodipalpis, comme Angelier, Decamps \& Rey (1963) l'ont montré sur le Céret
(Tarn) et Lavandier \& Dumas (1970) sur le ruisseau d'Agos (Htes-Pyrénées), - dont la température varie peu au cours de l'année.

\subsection{2. - Cycle d'Atractides gibberipalpis}

Les pourcentages de nymphes les plus élevés paraissent se situer en été aux altitudes moyennes et au printemps dans les eaux les plus froides (Couplan). En fait, le cycle n'est pas net: on trouve nymphes et adultes de l'été au début du printemps suivant, des $Q$ ovigères de l'hiver au début du printemps. Il faut noter que les récoltes de printemps correspondent au début de l'étiage sur les ruisseaux d'Ardengost et de Morère, mais avant le début de la fonte des neiges à Couplan, Eget et sur le ruisseau de Lavedan.

Le cycle de Sperchon denticulatus est analogue à celui d'Atractides gibberipalpis. Mais, en Suisse, Bader (1968) conclue à un cycle annuel dans le Jura, atteignant 1 an $1 / 2$ en altitude. Lavandier \& Dumas considèrent l'espèce comme annuelle.

Pour toutes les espèces, le synchronisme des différentes phase du cycle est moins net dans les mous. ses (où le rôle du courant et des crues est at ténué) que sous les pierres; moins net aussi sur les biefs dans lesquels la violence des crues est réduite (aval des retenues hydro-électriques).

\section{4. - Cycles et période d'étiage}

Chez Torrenticola similis, Protzia invalvaris, Sperchon clupeifer, Lebertia maglioi, Hygrobates calliger, Atractides nodipalpis, et beaucoup d'autres espèces 
des basses vallées (notamment Aturus scaber et Kongsbergia matema), le cycle débute au printemps ou en été, après la période des hautes eaux, alors que la température s'élève; il se termine à l'automne, alors que la température diminue et que commence la période des hautes eaux.

Atractides gibberipalpis, Sperchon denticulatus et nombre d'espèces de haute altitude, - Feltria notamment, - ont un cycle qui débute après la fonte des neiges. Il se poursuit en automne et durant l'hiver, c'est-à-dire durant la période d'étiage des cours d'eau à régime nival.

Ces deux types de cycles rappellent ce qu'on observe chez les Poissons : - Cyprinidés se reproduisant à la fin du printemps et alevins se développant au cours de l'été, durant l'étiage des rivières de plaine, - Salmonidés (sauf l'Ombre et la Truite arcen-ciel) se reproduisant à la fin de l'automne tandis que les alevins se développent durant l'hiver. Ces deux types de reproduction correspondent à deux régimes des eaux, à deux périodes d'étiage différentes.

\section{5. - Conclusions}

Le régime des eaux apparaît non seulement comme le synchronisateur des cycles biologiques des Hydracariens, mais aussi comme un facteur limitant dans la répartition des espèces.

C. Motas (1928) a montré que certaines espèces d'Hydracariens ne pénétraient pas dans les massifs centraux alpins, mais seulement dans les Préalpes. Ce sont notamment :

- Protzia rugosa

- Protzia brevipalpis

- Lebertia maglioi

- Torrenticola elliptica

- Feltria rouxi

- Aturus scaber

- Aturus asserculatus

- Alurus crinitus

- Kongsbergia materna

Atractides gibberipalpis, Feltria rubra, F. setigera, $F$. minuta vivent à la fois dans les Préalpes et les massifs centraux, tandis que Aturus elongatus vit seulement dans les massifs centraux.

Nous retrouvons cette opposition entre espèces de Mittelgebirge et de Hochgebirge, des auteurs de langue allemande, dans les Pyrénées: - dominance du genre Aturus, Torrenticola similis (homologue pyrénéen de $T$. elliptica), Lebertia maglioi, Atractides nodipalpis, dans les cours d'eau du piémont pyrénéen.

- dominance du genre Feltria, Atractides gibberipalpis, Sperchon denticulatus,... dans les cours d'eau de la chaîne axiale, à fonte des neiges tardive et étiage d'hiver.

Les deux types biologiques d'Hydracariens mis en évidence dans les Alpes comme dans les Pyrénées correspondent à deux types de régime des eaux : nival et nival de transition d'une part, et pluvial et pluvio-nival d'autre part. A ces deux types biologiques, il faut ajouter les Torrenticola et quelques espèces comme Pseudotorrenticola rhynchota, abondants dans les rivières méditerranéennes à étiages d'été prononcés.

Si nous considérons maintenant une rivière, de l'amont vers l'aval, elle présente, le long de son profil d'équilibre :

- une zone à forte pente, où les galets sont constamment entraînés ; les mousses peuvent recouvrir roche-mère et gros blocs de rochers, assurant la continuité dans le temps des biotopes à Hydracariens. - une zone à substrats de galets instables, de fonds remaniés à chaque crue.

- une zone où l'épaisseur des alluvions déposées par la rivière est importante. L'horizon superficiel est instable, mais la continuité dans le temps du biotope est assurée en profondeur.

La succession amont-aval des espèces, sur une rivière, correspond en fait à la succession de ces trois zones. Dans la zone instable vivent les espèces pionnières (Lebertia, Sperchon,...), à taux de reproduction important. Les espèces d'un mème genre se sont diversifiées en colonisant les biotopes disponibles et se remplacent en fonction du courant et de la température.

Dans les mousses,et le milieu hyporhéique, où la continuité dans le temps du subst rat est assurée, le taux de reproduction des espèces est plus faible. Lorsque les ressources nutritives sont diversifiées, plusieurs espèces d'i - même genre (Feltria, Aturus,...) peuvent cohabiter (dans les mousses). Dans le milieu hyporhéique, les seules ressources nutritives sont des détritus apportés par l'eau, et on n'observe généralement qu'une espèce par genre. 
Nous pouvons maintenant établir une correspondance entre les associations d'Hydracariens et les types biologiques mis en évidence :

a) Cours d'eau à régime nival :

- zone des mousses : association II, à Feltria

- zone d'instabilité : association III (Atractides gibberipalpis et Sperchon denticulatus), puis association V (Sperchon hispidus, Lebertia maglioi, Torrenticola similis et Atractides nodipalpis).

— milieu hyporhéique : $T$, similis et espèces hyporhéiques strictes.

b) Cours d'eau à régime pluvio-nival et pluvial :

- zone de mousses : association IV (Aturus et Kongsbergia)

- zone d'instabilité : association VI (Sperchon clupeifer, Sperchonopsis verrucosa, Torrenticola similis, Atractides nodipalpis), puis association VII (Torrenticola anomala et $T$. madritensis).

- milieu hyporheique : Torrenticola et espèces hyporhéiques strictes.

c) Cours d'eau des Coteaux de Gascogne :

- zone des mousses non définie

- zone d'instabilité : associations VII et VIII (Lebertia porosa, Hygrobates calliger,... et Torrenticola).

- milieu hyporhéique : Torrenticola et espèces hyporhéiques strictes.

Certaines associations correspondent pratique. ment à un seul type biologique (association III, par exemple). D'autres réunissent plusieurs types biologiques, et on peut parler de spectre biologique. Ainsi, le peuplement de la basse vallée d'une rivière à régime nival peut être constitué à la fois par des espèces de régime pluvio-nival qui ont le temps de se développer entre juillet et l'automne, et des espèces de régime nival qui poursuivent leur développement durant l'hiver.

En aval d'une retenue hydro-électrique, en montagne, sur un bief de rivière à débit réservé et crue réduite, des espèces de régime pluvio-nival ou pluvial viennent s'ajouter aux espèces de régime nival (Neste d'Aure à Couplan et Eget, ruisseau de Lavedan).

Les paramètres topographiques et hydrologiques sont bien représentatifs des facteurs écologiques, mais ils ne suffisent pas, seuls, à expliquer le peu. plement des eaux courantes. La continuité dans le temps du biotope est un facteur écologique fondamental

Enfin, aucun facteur écologique ne rend compte véritablement du peuplement des eaux à faible courant (sources limnocrènes,,.. ) en haulte altitude, et il faut faire appel à l'historique du peuplement. Un certain nombre d'especes sont des relictes glaciaires: Hydrovolzia placophora, L. dubia, L. zschokkei, Rivobates norvegicus, Feltria minuta,... Hydryphantes pyrenaicus, proche d'une espece sibérienne, peut aussi se rat tacher à ce groupe. Ces espèces ont colonisé en haute altitude des biotopes équivalents à ceux qu'elles occupaient à un niveau plus bas durant les glaciations. Mais, en haute altitude, ces biotopes sont réduits, morcelés (mares, sources limnocrènes, petits biefs de ruisseaux à faible courant). L'association I ne s'insère pas dans la succession logique des associations le long d'un cours d'eau.

\section{6. - Structure des communautés d'Hydracariens}

\section{1. - Indices biospécifiques}

Nous avons utilisé trois indices pour caractériser la structure des communautés d'Hydracariens :

- Indice de diversité de Fischer, $\mathrm{S}=\alpha \log _{\mathrm{e}}(1+$ $\mathrm{N} / \alpha$ ), ou $\mathrm{Set} \mathbf{N}$ désignent le nombre d'espèces et d'individus et $\alpha$ l'indice de diversité.

- Indice de dominance de Simpson, $\mathrm{x}\left(\mathrm{N}_{\mathrm{i}} / \mathrm{N}\right)^{2}$, où $\mathrm{N}_{\mathrm{i}}$ et $\mathbf{N}$ désignent respectivement le nombre d'individus de l'espèce $i$ et le nombre total d'individus.

— Indice E/G (nombre d'espèces/nombre de genres).

Ce dernier indice rend compte de deux règles de biocoenotique de Thienemann (1939):

- les biotopes aux conditions ambiantes défavorables comportent un grand nombre de genres avec chacun peu d'espèces.

- les biotopes aux conditions ambiantes favorables comportent un nombre réduit de genre avec chacun un grand nombre d'espèces.

Globalement (tableau V), les trois indices paraissent suivre le facteur amont-aval. L'indice de diversité et le rapport $\mathbf{E} / \mathbf{G}$ s'élèvent de la source au cours inférieur, tandis que l'indice de Simpson tend vers 0 . A altitude égale, la diversité est plus élevée dans les mousses que sous les galets. $\alpha$ et E/G élevés et $\mathrm{D}$ faible paraissent liés à la continuité dans le temps 
Tableau V. Evolution amont-aval des indices Fisher ( $\alpha$ ), de Simpson (D) et du rapport Especes/Genres.

\begin{tabular}{|c|c|c|c|c|c|c|c|c|}
\hline & STATION AMONT & $\alpha$ & $\mathrm{D}$ & EG & STATION AVAL & $\alpha$ & $\mathrm{D}$ & $\mathrm{E} G$ \\
\hline $\begin{array}{l}\text { Substrats de mousses: } \\
\text { - Arros }\end{array}$ & 81 & 2 & 0.75 & 1,1 & 96 & 3,2 & 0,17 & 1 \\
\hline $\begin{array}{l}\text { - Esqueda (aff. Arros) } \\
\text { - Saux } \\
\text { - Arsoué } \\
\text { - Louron } \\
\text { - Bareille } \\
\text { - Ardengost } \\
\text { - Beyrède } \\
\text { - Nistos } \\
\text { - Camoudiet } \\
\text { - Hourc }\end{array}$ & $\begin{array}{r}84 \\
6 \\
13 \\
32 \\
42 \\
39 \\
63 \\
75 \\
99 \\
103\end{array}$ & $\begin{array}{l}3 \\
3,1 \\
1 \\
2,1 \\
4,3 \\
7 \\
2,5 \\
2,5 \\
3 \\
2,5\end{array}$ & $\begin{array}{l}0,82 \\
0,19 \\
0,57 \\
0,43 \\
0,2 \\
0,1 \\
0,45 \\
0,41 \\
0,38 \\
0,4\end{array}$ & $\begin{array}{l}1,3 \\
1,3 \\
1,2 \\
1 \\
1,2 \\
1 \\
1,17 \\
1,27 \\
1 \\
1,2\end{array}$ & $\begin{array}{c}96 \text { (Arros) } \\
19 \\
72 \text { (Lortet) } \\
61 \\
58 \\
65 \\
69 \\
77 \\
102 \\
104\end{array}$ & $\begin{array}{l}3,2 \\
4,2 \\
5 \\
2,7 \\
4,3 \\
2,8 \\
2,5 \\
3 \\
3,2 \\
2,9\end{array}$ & $\begin{array}{l}0,17 \\
0,18 \\
0,06 \\
0,2 \\
0,2 \\
0,1 \\
0,22 \\
0,38 \\
0,01 \\
0,43\end{array}$ & $\begin{array}{l}1 \\
1,1 \\
1,5 \\
1,28 \\
1,12 \\
1,5 \\
1,14 \\
1,2 \\
1 \\
1\end{array}$ \\
\hline \multicolumn{9}{|l|}{ Substrats de galets: } \\
\hline $\begin{array}{l}\text { - Arros } \\
\text { - Badet } \\
\text { - Moudang } \\
\text { - Louron }\end{array}$ & $\begin{array}{l}80 \\
11 \\
43 \\
33\end{array}$ & $\begin{array}{l}1,5 \\
1,8 \\
1 \\
1\end{array}$ & $\begin{array}{l}0,48 \\
0,34 \\
0,48 \\
0,56\end{array}$ & $\begin{array}{l}1,25 \\
1,5 \\
1,2 \\
1\end{array}$ & $\begin{array}{c}95 \\
44 \text { (Eget) } \\
59 \text { (Grézian) } \\
60\end{array}$ & $\begin{array}{l}3,2 \\
2,7 \\
3 \\
2,8\end{array}$ & $\begin{array}{l}0,28 \\
0,29 \\
0,15 \\
0,24\end{array}$ & $\begin{array}{l}2 \\
1,8 \\
2,2 \\
2,3\end{array}$ \\
\hline $\begin{array}{l}\text { Moyenne des stations à } \\
\text { substrats de mousses : }\end{array}$ & Amont & 2.75 & 0.34 & 1,2 & Aval & 3,4 & 0,17 & 1,17 \\
\hline $\begin{array}{l}\text { Moyenne des stations à } \\
\text { substrats de galets: }\end{array}$ & Amont & 1,3 & 0,55 & 1,25 & Aval & 2,9 & 0,24 & 2 \\
\hline $\begin{array}{l}\text { Moyenne des stations de } \\
\text { la vallée d'Aure: }\end{array}$ & $\begin{array}{l}\text { Au-delà de } \\
1800 \mathrm{~m}\end{array}$ & 2,15 & 0,4 & 1,3 & $\begin{array}{l}\text { Au-dessous } \\
\text { de } 1800 \mathrm{~m}\end{array}$ & 4 & 0,24 & 1,15 \\
\hline $\begin{array}{l}\text { Moyenne des stations de } \\
\text { l'Adour }\end{array}$ & 9 stations & 2.3 & 0,31 & 1,17 & & & & \\
\hline $\begin{array}{l}\text { Moyenne des stations des } \\
\text { Baronnies: }\end{array}$ & 19 stations & 2,6 & 0,4 & 1,3 & & & & \\
\hline $\begin{array}{l}\text { Moyenne des stations du } \\
\text { Gers : }\end{array}$ & 7 stations & 4,7 & 0.19 & 1,6 & & & & \\
\hline
\end{tabular}

du biotope, à la présence de quelques genres (Torrenticola, Feltria, Aturus) qui peuvent être représentés par plusieurs espèces dans chaque station.

En fonction de leurs indices biospécifiques, les stations prospectées peuvent être regroupées en trois catégories :

- les stations à peuplement de type pionnier. Nombre moyen d'espèces : 5,8, $-\alpha$ moyen : 1,2. $-\mathrm{D}$ moyen : 0,7. $-\mathrm{E} / \mathrm{G}$ moyen : 1,2. Ces stations sont des sources ou des ruisseaux de faible numéro d'ordre. En fait, ces indices moyens ne correspondent pas exactement à la réalité, car nous avons éliminé des analyses 13 stations comportant moins de 3 espèces.

- les stations à peuplement de type climacique. Nombre moyen d'espèces : $10 .-\alpha$ moyen : 2,5 .
D moyen : 0,14. - E/G moyen : 1,5. Ce sont 20 stations de mousses ou de galets de basse altitude.

- entre ces deux extrêmes, une quarantaine de stations intermédiaires. Nombre moyen d'espèces : 8,16. $-\alpha$ moyen : 1,5 . $-\mathrm{D}$ moyen : 0,4 . $-\mathrm{E} / \mathrm{G}$ moyen : 1,3 .

\section{2. - Diagrammes rangs-fréquences en fonction du type de peuplement}

Pour les trois types de peuplement mis en évidence par les indices biospécifiques (tableau V), nous avons établi un diagramme rang-fréquence (Frontier 1977 a et b). La fréquence relative de chaque espèce (pourcentage de la population) est portée en 

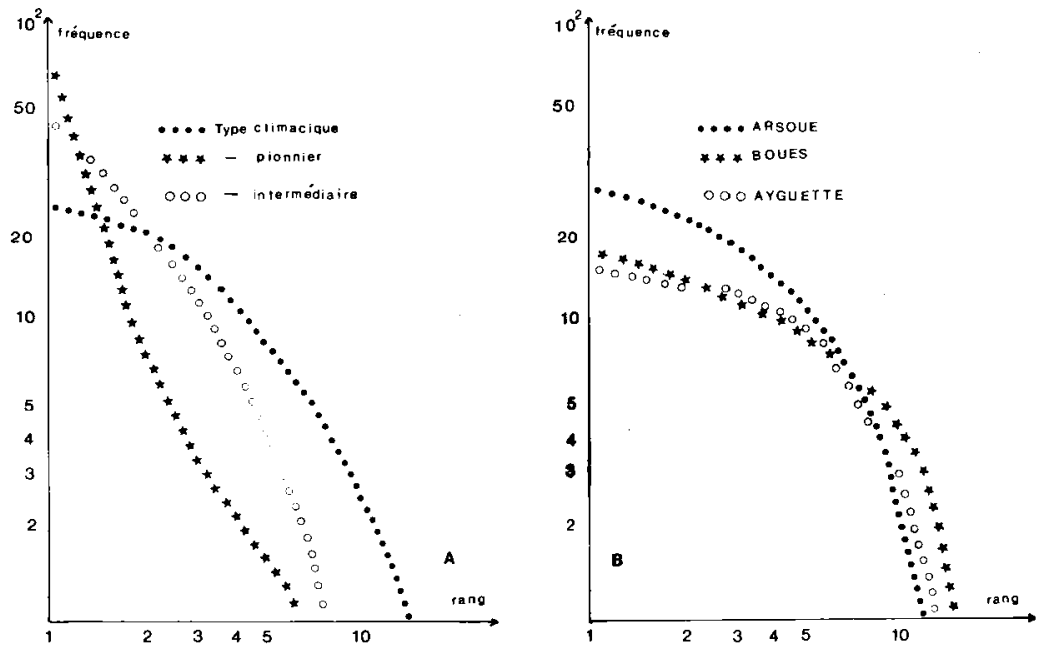

Fig. 14. Diagramme rang-fréquence. $14 \mathrm{~A}$ : Diagramme correspondant à la moyenne des peuplements pionniers, climaciques et intermédiaires. $14 \mathrm{~B}$ : Diagramme correspondant au peuplements d'Arsoué (st. 16), de l'Ayguette (st. 89) et du Bouès (st. 94).

ordonnée, et son rang (par ordre décroissant de fréquence) en abcisse ( $f \mathrm{ig} .14$ : échelle logarithmique en abcisse et en ordonnée).

La courbe correspondant aux peuplements pionniers a une allure concave vers le haut, dans sa partie gauche, - indiquant la prédominance d'un petit nombre d'espèces. Frontier décrit ensuite une inflexion de la courbe, qui devient convexe dans sa partie droite. Cette inflexion est à peine marquée (fig. $14 \mathrm{~A}$ ). La courbe correspondant aux peuplements dits climaciques est entièrement convexe, tan dis que celle correspondant aux peuplements intermédiaires se situe entre les deux précédentes.

Mandelbrot (1953) a montré que le problème des alignements remarquables des mots d'une langue dans les diagrammes rang-fréquence se ramenait au problème de l'adaptation d'un code à un canal d'information, - de façon à ce que la quantité d'information transmise par unité de temps ou unité de coût soit maximale. Lorsque tous les symboles ont le même coût, l'information maximale est obtenue par leur équifréquence ; lorsqu'ils ont des coûts différents, les plus coûteux sont les plus rares. Maldelbrot en déduit la loi rang-fréquence des symboles: $p_{n}=P(n+B)^{-} \gamma ;$ la courbe est asymptote à la droite $\log p-\log n$, où $p_{n}$ est la fréquence du symbole de rang $n$. $P$ varie avec le nombre de symboles ( $\mathrm{P}=100$ lorsqu'on l'exprime en fréquence relative) : $\mathbf{B}$ détermine le décollement de la courbe sous l'asymptote, en ordonnée ; $\gamma$ correspond à la pente de l'asymptote.

Les diagrammes rang-fréquence établis pour le phytoplancton des lacs d'Auvergne (Devaux 1976), le microplancton du golfe de Marseille (Travers 1971), des Ptéropodes (Frontier 1974) ou des Chaetognathes (Frontier \& Bour 1976), sont sensiblement rectilignes, - c'est-à-dire avec de faibles valeurs de B. Ils correspondent à la présence d'espèces dont le coût (ici, la taille) est très différent : les espèces les plus grandes sont les plus rares. La pente de la 


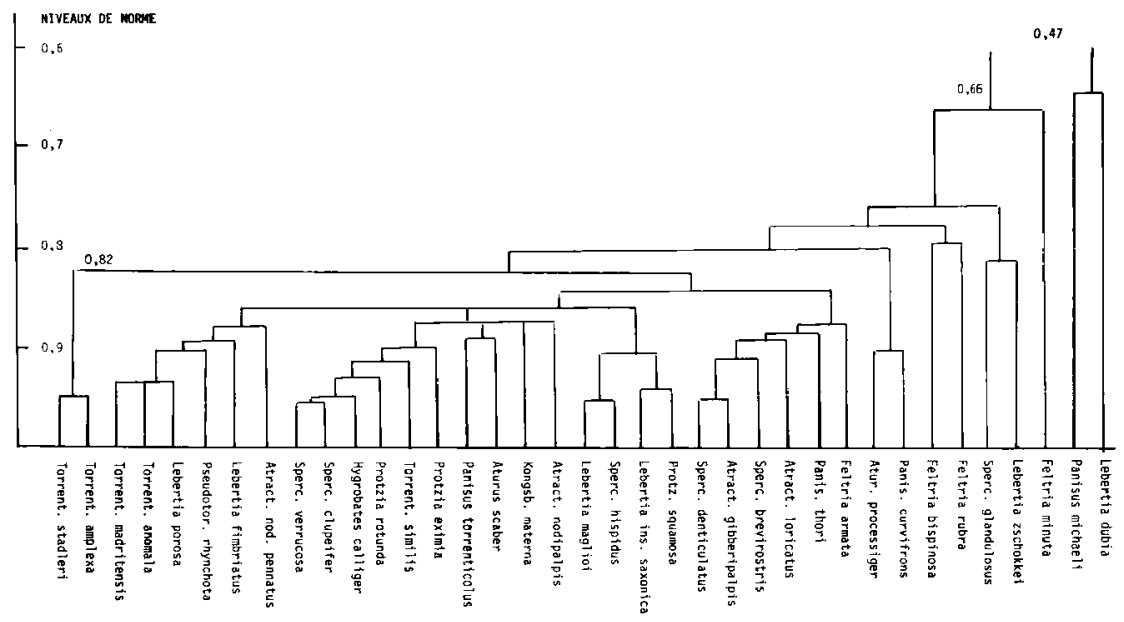

Fig. 15. Hiérarchisation des espèces sur les cinq premiers axes.

courbe se rapproche de l'optimum décrit par Mandelbrot pour la linguistique : $\gamma=1$ et $\mathbf{B}$ voisin de 0 .

Pour la moyenne des peuplements en Hydracariens de type climacique, la courbe obtenue correspond à la relation

$$
P_{n}=100(n+1,5)-1,6
$$

La pente $(\gamma=1,6)$ est proche de la pente optimum de Mandelbrot. Mais B plus élevé $(B=1,5)$ traduit une certaine équifréquence des espèces au stade climacique. B reflète probablement la présence de genres comme Feltria, Aturus, Torrenticola, comportant chacun plusieurs espèces de tailte sensiblement égale. La valeur de $\mathrm{B}$ pourrait caractériser les taxocoenoses d'Hydracariens dans les peuplements climaciques.

\section{3. - Hiérarchisation des espèces à partir de l'analyse factorielle des correspondances (fig. 15)}

La hiérarchisation des espèces tirée des cinq pre. miers axes de l'analyse factorielle des correspondances montre des corrélations étroites entre celles-ci, - à basse altitude et dans les mousses, - et des corrélations beaucoup plus lâches en haute altitude (même dans les mousses).

La presque totalité des espèces des associations VII et VIII (Coteaux de Gascogne) sont corrélées à des niveaux égaux ou supérieurs à $0,9 \%(0,96$ pour Torrenticola stadleri et $T$. amplexa). Les associations de haute altitude apparaissent par contre à des niveaux de liaison de 0,66 à 0,81 .

Il est intéressant de noter à quels niveaux se 
regroupent entre elles les différentes associations. L'ensemble des espèces de haute altitude constitue un peuplement isolé, ne se regroupant aux autres associations qu'au niveau 0,78 (Feltria rubra et $F$. bispinosa), 0,76 (Lebertia zschokkei et Sperchon glandulosus), 0,66 (Feltria minuta) et même 0,47 (Lebertia dubia et Panisus michaeli). L'isolement de l'association I par rapport aux autres associations (5.4) est ainsi vérifié.

Un ensemble de 6 espèces (association III), - Panisopsis thori, Sperchon denticulatus, Sp. brevirostris, Atractides gibberipalpis, $A$. loricatus et Feltria amnata, $\rightarrow$ ne se regroupe aux autres qu'au niveau 0,84 . Ce sont les espèces des massifs centraux, aux étiages d'hiver. De mêrne, Torrenticola amplexa et $T$. stadleri (regroupement avec les autres espèces au niveau 0,82 ) sont des espèces isolées caractéristiques des eaux à débit estival réduit.

Enfin, Panisopsis curvifrons et Aturus elongatus processiger sont également plus isolées que ne laisse prévoir leur appartenance à l'association IV. En fait, elles sont plus sténothermes que les autres espèces de l'association et se retrouvent, sans être jamais abondantes, à des altitudes plus élevées.

\section{4. - Conclusions}

La stabilité dans le temps du substrat se traduit par une structuration du peuplement, avec des espèces à taux de reproduction plus faible et la présence simultanée de plusieurs espèces par genre. L'indice de Fisher élevé, l'indice de Simpson proche de 0, rendent compte de la diversité des espèces dans les biotopes à continuité dans le temps assurée.

Les diagrammes rang-fréquence suggèrent une * gestion de l'information interne en fonction d'un coût attaché à chaque espèce " (Frontier 1976). Un coût correspondant à la taille est en fait un coût énergétique. Mais il ne faut pas oublier que la dimension des feuilles des mousses et le diametre des interstices limitent la taille des espèces muscicoles et hyporhéiques. L'équifréquence constatée dans les peuplements à large continuité temporelle est traduite par la valeur élevée de $B$, qui pourrait caractériser les taxocoenoses d'Hydracariens.

Enfin, la hiérarchisation des espèces confirme l'isolement de l'association I dans la succession amont-aval.

\section{7. - Équilibre dynamique des peuplements d'eaux courantes}

\section{1. - Stations à large continuité spatio-temporelle}

Une structuration croissante des taxocoenoses, de I'amont vers l'aval, a été mise en évidence par les indices de diversité et de dominance, le rapport $E / G$ et les diagrammes rang-fréquence. Mais ces indices ont été calcu lés sur la station la plus élevée et la plus basse de chaque cours d'eau (tableau V). Nous avons négligé des stations intermédiaires à diversité parfois très élevée et également éliminé des analyses les stations comportant moins de 3 especes.

La majeure partie de ces dernières stations, à faible diversité, est située au niveau de l'étage montagnard, lorsque la pente s'accentue et entraîne une trop grande instabilité du substrat de galets (association III). Sperchon denticulatus et Atractides gibberipalpis sont les dernières espèces qui subsistent, avant la disparition de la faune pétricole. Les autres stations comportant également moins de 3 espèces sont des biotopes de surface réduite (sources,...).

Une surface de mousses importantes, à $1100 \mathrm{~m}$ d'altitude, en courant lent à vif, sur le ruisseau de la Mousquère (Arsoué, st, 16) héberge 12 espèces, dont 4 appartiennent au seul genre Felfria :

\begin{tabular}{lr} 
Feltria rubra & $25 \%$ \\
Sperchon denticulatus & $21 \%$ \\
Feltria amata & $19 \%$ \\
Atractides gibberipalpis & $10 \%$ \\
Torrenticola similis & $6 \%$ \\
Feltria minuta & $5 \%$ \\
Feltria bispinosa & $4 \%$ \\
Atractides loricatus & $3 \%$ \\
Protzia invalvaris & $2 \%$ \\
Panisus michaeli & \\
Torrenticola anomala & $5 \%$ \\
Hydrovolzia placophora & ) \\
\hline
\end{tabular}

L'indice de diversité ( $\alpha=4)$, l'indice de Simpson $(\mathrm{D}=0,13)$ indiquent l'absence de dominance. $\mathrm{E} / \mathrm{G}$ $=1,7$. Les 12 espèces appartiennent à 3 associations différentes (associations I, II et III).

A $2080 \mathrm{~m}$, sur une surface importante d'Hypnacées du ruisseau d'Estarragne, Mattei (1966) a recueilli 7 espèces :

$\begin{array}{ll}\text { Feltria armata } & 31 \% \\ \text { Feltria bispinosa } & 25 \%\end{array}$




$\begin{array}{lr}\text { Feltria minuta } & 18 \% \\ \text { Feltria quadrispinosa } & 12 \% \\ \text { Feltria rubra } & 10 \% \\ \text { Lebertia (2 espèces) } & 4 \%\end{array}$

La diversité est faible; mais une équifréquence relative des espèces, un rapport $E / G$ egal à 3,5 traduisent cependant, malgré l'altitude, l'évolution vers un peuplement de type climacique. Ceci avec un groupe d'espèces du genre Feltria muscicoles, caractéristiques du régime des eaux nival.

Au niveau de l'étage montagnard, lorsque la pente des cours d'eau s'accentue, les substrats sont variés, des graviers aux blocs de rochers, mais morcelés. C'est la zone de contact entre les associations II (mousses) et III (mousses et galets). Dans les mousses, $\alpha$ est élevé, mais D traduit la dominance de quelques espèces à fort taux de reproduction (nymphes de Sperchon denticulatus et Atractides gibberipalpis, notamment) :

- sur la Neste de Couplan (st. 28), 16 espèces vivent dans les mousses, mais 5 seulement représentent $90 \%$ des individus ( $\alpha=3,8 ; \mathrm{D}=0,59$ ).

- dans des mousses de la Neste d'Aure à Eget (st. $45), 15$ espèces, dont 4 représentent $90 \%$ des individus $(\alpha=2 ; \mathrm{D}=0,33$ )

- dans des mousses sur le ruisseau de Lavedan (st. 48), 14 espèces, dont 3 représentent $75 \%$ des individus ( $\alpha=3 ; \mathrm{D}=0,23$ ).

Mais aux mêmes niveaux, le peuplement des galets est pratiquement réduit à Sperchon denticulatus et Atractides gibberipalpis (association III).

Sur des cours d'eau affluents de la Neste d'Aure, dans les Baronnies et sur les Coteaux de Gascogne, un certain nombre de stations sont aussi caractérisées par une diversité exceptionnelle, supérieure à la diversité moyenne (tableau $\mathrm{V}$ ) : ainsi les stations 78 (Nistos), 89, (Ayguette) ou 94 (Bouès).

NISTOS (station 78, galets). -13 especes

Torrenticola similis

Lebertia fimbriata

Torrenticola anomala

Protzia invalvaris $\%$ individus

$37 \%$

$27 \%$

$8 \%$

6. $\%$
Torrenticola madritensis

Sperchon clupeifer

Lebertia insignis

Hygrobates nigromaculatus

Atractides nodipalpis

Hygrobates calliger

Sperchonopsis verrucosa

Pseudotorrenticola rhynchota

Sperchon hispidus
$5 \%$

$4 \%$

$3 \%$

$3 \%$

$1,5 \%$

$1,5 \%$

$1,5 \%$

$1,5 \%$

$1 \%$

$\alpha=3 .-\mathrm{D}=0,2 .-\mathrm{E} / \mathrm{G}=1,8$

AYGUETTE (station 89, mousses). -23 espèces.

$\begin{array}{lcr}\text { Sperchon clupeifer } & 16 & \% \\ \text { Torrenticola similis } & 14 & \% \\ \text { Hygrobates fluviatilis } & 14 & \% \\ \text { Atractides nodipalpis } & 12 & \% \\ \text { Atractides gibberipalpis } & 9,5 \% \\ \text { Protzia invalvaris } & 4,2 \% \\ \text { Lebertia maglioi } & 4,2 \% \\ \text { Sperchon denticulatus } & \\ \quad \text { hibernicus } & 3,5 \% \\ \text { Lebertia porosa } & 3,1 \% \\ \text { Torrenticola anomala } & 3 & \% \\ \text { Kongsbergia materna } & 2 & \% \\ \text { Sperchonopsis verrucosa } & 2 & \% \\ \text { Hygrobates calliger } & 1,4 \% \\ \text { Protzia rontunda } & 0,7 \% \\ \text { Panisus torrenticolus } & 0,7 \% \\ \text { Atractides tener } & 0,7 \% \\ \text { Aturus crinitus } & 0,7 \% \\ \quad 5 \text { especes } & 2,3 \% \\ & \end{array}$

Cette liste confirme le fait que l'opposition espèces muscicoles-espèces pétricoles s'estompe à basse altitude.

BOUES (Station 94, galets). 14 espèces.

$\begin{array}{lrl}\text { Torrenticola amplexa } & 17 & \% \\ \text { Torrenticola brevirostris } & 12 & \% \\ \text { Lebertia maglioi } & 11 & \% \\ \text { Torrenticola similis } & 9 & \% \\ \text { Torrenticola madritensis } & 9 & \%\end{array}$




$\begin{array}{ll}\text { Torrenticola stadleri } & 8,5 \% \\ \text { Hygrobates calliger } & 8,5 \% \\ \text { Atractides nodipalpis } & \\ \quad \text { pennatus } & 6 \% \\ \text { Lebertia porosa } & 3,5 \% \\ \text { Sperchon clupeifer } & \\ \begin{array}{l}\text { Pseudotorrenticola } \\ \text { rhynchota }\end{array} & \\ \text { Atractides lunipes } & , \\ \text { Woolastookia rotundifrons } & \\ \alpha+6 .-\mathrm{D}=0,04 .-\mathrm{E} / \mathrm{G}=2 . & \\ \alpha\end{array}$

Trois stations seulement, 16 (Arsoué), 89 (Ayguette) et 94 (Boués) représentent 39 des 82 espèces recueillies sous les pierres ou dans les mousses.

Avec 4 stations (les trois précédentes et la st. 28 (Couplan), 46 espèces sur 82 .

Avec 5 stations (les quatre précédentes et la st. 78 , (Nistos), 49 espèces sur 82.

Dans chacune de ces cinq stations figurent des espèces appartenant à plusieurs associations. Les huit associations mises en évidence par l'analyse factorielle des correspondances sont regroupées en 3 stations seulement :

- st. 16 (Arsoué) associations I, II et III

- st. 89 (Ayguette) associations IV, $V$ et VI

- st. 94 (Bouès) associations VI, VII et VIII.

Les cinq stations citées sont situées à des altitudes très différentes ( 300 à $1380 \mathrm{~m}$ ), un régime des eaux de type nival, pluvio-nival ou pluviothermique, des temperatures estivales entre 8 et $16^{\circ} \mathrm{C}$, des substrats de mousses ou de galets. Mais elles ont en commun :

- la largeur du lit (2 à $5 \mathrm{~m}$ )

- un long bief présentant un substrat homogène (quelques dizaines à quelques centaines de mètres) - une épaisseur importante d'alluvions pour les substrats de galets.

Tous ces caractères assurent la continuité dans l'espace du biotope.

- une pente faible (1 à $3 \%$ )

- des crues réduites, érodant peu le substrat.

Ces caractères assurent la stabilité, la continuité dans le temps du biotope.

La structuration croissante des taxocoenoses d'Hydracariens, de l'amont vers l'aval, ne traduit pas seulement une réduction des espèces avec l'altitude. Sur un cours d'eau, de l'aval vers l'amont, la surface des biotopes se réduit, et on tend vers une mosaïque de substrats de petites dimensions, aux facteurs écologiques de plus en plus accentués, qui sélectionnent un type biologique bien défini.

Mais, à toute altitude, lorsqu'un biotope présente une surface et une continuité dans le temps importantes, la diversité spécifique et la structuration des taxocœenoses s'accroissent.

Le facteur surface, dans les analyses factorielles des correspondances, a été pris en compte seulement pour les mousses. Le facteur continuité tem. porelle a été évoqué de façon indirecte, par l'intermédiaire de la pente. Quant à la violence des crues, facteur d'érosion du substrat et de destruction des organismes benthiques, il a rarement pu être évalué.

\section{2. - Equilibre dynamique des peuplements}

De l'analyse factorielle des correspondances, nous avons tiré un classement des espèces suivant une succession amont-aval (3.1.). Ce classement ne correspondait pas entièrement à la réalité, et nous avons reconnu des types biologiques d'Hydracariens (en fonction d'accomodements à trois types de substrats et trois types de régime des eaux). On peut ainsi définir le peuplement d'une station par un spectre biologique, - pourcentage des différents types biologiques en fonction d'un complexe stationnel. La continuité du biotope dans l'espace et dans le temps fait partie du complexe stationnel. La continuité spatiale a une influence sur la structure de la taxocanose ; la continuité temporelle pourrait déterminer la stratégie ( $r$ ou $k$ ) de reproduction des espèces.

Les modalités du peuplement des eaux courantes par les Hydracariens rappellent celles du peuplement des mousses corticoles par les Thécamoebiens. Selon Bonnet (1973), " la colonisation des mousses du sol est aisée, car les biotopes sont plus ou moins continus et leur continuité dans le temps bien assurée. De plus, elles sont en relation directe avec le réservoir inépuisable d'individus que constitue le sol. Une fraction du biotope détruite accidentellement est rapidement recolonisée, et, lorsqu'il n'y a pas de facteurs écologiques extrêmes, les mousses du sol présentent une grande diversité spécifique. Le peuplement des mousses corticoles est par contre sujet à de nombreux aléas : biotopes discontinus, 
dessication rapide, colonisation à partir du sol malaisée,,.. La colonisation du biotope par de nouvelles espèces est difficile et fluctuante, - surtout lorsque les arbres sont isolés. Le caractère problématique de la colonisation des mousses corticoles a pour conséquence une indépendance entre la diversité spécifique et le caractère plus ou moins favorable des facteurs écologiques \#.

Le peuplement des mousses corticoles par les Thécamoebiens et des eaux courantes par les Hydracariens pose des problèmes identiques à ceux que $\mathrm{Mac}$ Arthur \& Wilson (1963) et Mac Arthur (1967) ont abordé à propos du peuplement des îles :

- une communauté insulaire est en équilibre dynamique, - les extinctions locales étant en moyenne compensées par des immigrations de la même espèce ou d'autres espèces.

- la diversité spécifique est fonction de la surface du territoire.

- le taux d'immigration diminue lorsque s'accroît la distance entre l'île et le réservoir d'espèces que représente le continent et les autres îles.

Le modèle d'équilibre de Mac Arthur \& Wilson s'applique également aux "îles virtuelles", - biotopes isolés comme un lac au milieu des terres, une source, un bief à courant lent sur une rivière à courant rapide,... Si la diversité spécifique diminue avec la surface du biotope, le peuplement des "îles virtuelles " de très petite surface doit être à la limite aléatoire, et celà d'autant plus que les biotopes sont éloignés les uns des autres.

La continuité temporelle des sources eșt assuruée, mais non leur continuité spatiale; elles constituent de véritables îles. Sur les 32 espèces d'Hydracariens recensées par Motas (1928) des sources du Dauphiné, 17 n'ont été citées qu'une scule fois ; 5 espèces, 2 fois ; 3 espèces, 3 fois. Les espèces les plus citées sont Sperchon denticulatus (11 fois), Lebertia zschokkei ( 9 fois), Lebertia tuberosa et Feltria minuta ( 8 fois), et $S p$. glandulosus, (7 fois), c'est-à-dire des espèces appartenant aux associations I et II (mousses et galets), qui ne caractérisent pas seulement les sources, mais aussi le cours supérieur des cours d'eau à régime nival. Les espèces strictement liées aux sources sont rares.

La faible surface des sources a pour conséquence une faible diversité spécifique, et leur isolement limite l'immigration des espèces strictement inféodées à ce biotope. La distribution de ces espèces devient aléatoire. Le phénomène de dérive, à ce niveau, joue plus en faveur de l'émigration que de l'immigration.

L'immigration par dérive joue un rôle plus important dans les stations des cours moyens et inférieurs. C'est peut-être la raison pour laquelle l'analyse factorielle des correspondances basée sur le seul critère présence-absence n'a pas permis de différencier les profils de chaque station d'un profil moyen (à l'exception, précisément, des stations de haute altitude et des Coteaux de Gascogne, isolées écologiquement et géographiquement) : il existe dans chaque station quelques espèces, représentées par très peu d'individus, et dont le type biologique ne correspond pas obligatoirement au complexe stationnel. Leur présence est probablement liée à une immigration par la dérive et leur survie peut être aléatoire. On retrouve ainsi, dans la basse vallée de la Neste d'Aure, après la fonte des neiges, des espèces de la haute vallée qui disparaissent ensuite.

Mais, en utilisant les critères d'abondance dans les analyses, nous avons retiré leur poids aux espèces faiblement représentées. En outre, plus de la moitié des espèces récoltées ont été éliminées des analyses parce-qu'elles figuraient dans deux stations au plus.

Un certain nombre de ces espèces ne figurant pas dans les analyses sont liées aux sources, et leur distribution est de caractère plus ou moins aléatoire :

\section{Hydrovolzia placophora \\ Hydrovolzia cancellata \\ Trichothyas petrophila \\ Lebertia stigmatifera \\ Hygrobates norvegicus \\ Atractides nodipalpis fonticolus \\ Atractides tener \\ Atractides vaginalis \\ Atractides lunipes,...}

D'autres espèces vivent dans le milieu hyporhéique, - de façon stricte ou préférentielle, et leur présence dans l'horizon superficiel des galets est inhabituelle :
Wandesia thori
Torrenticola fagei
Torrenticola remyi
Atractides latipalpis
Atractides pumilus
Ljania bipapillata
Woolastookia rotundifrons
Kongsbergia clypeata 
Certaines sont endémiques pyrénéennes :

Hydryphantes pyrenaicus

Torrenticola fagei

Feltria quadrispinosa

Aturus prenanti

Aturus villosus

Enfin, quelques espèces sont, dans les Pyrénées, à la limite sud de leur aire de répartition :

Lebertia sefvei

Lebertia salebrosa

Feltria rouxi

Aturus spatulifer

Kongsbergia ruttneri

tandis que Lebertia lusitanica est une espèce connue d'Espagne et Atractides subasper une espèce commune sur le littoral méditerranéen et toujours rare ailleurs.

La dispersion des Hydracariens s'effectue par deux voies différentes:

- les larves sont parasites externes des imagos d'Insectes à larves aquatiques. La dispersion peut se faire dans toutes les directions.

- nymphes et adultes dérivent avec le courant, vers l'aval. Cette dérive affecte surtout les espèces de l'horizon superficiel des galets.

Nous ne connaissons pas la capacité de dispersion des larves d'Hydracariens; elle doit dépendre de la durée du stade, et de la capacité de dispersion de l'hôte. Mais le nombre élevé d'espèces représentées par quelques individus dans un nombre restreint de stations suggère un taux d'immigration beaucoup plus limité pour les Hydracariens des sources, muscicoles ou hyporhéiques que pour les Hydracariens pétricoles.

En résumé, l'équilibre dynamique des taxocœnoses d'Hydracariens est réalisé avec un nombre élevé d'espèces dans les stations à large continuité spatio. temporelle, - surfaces importantes d'Hypnacées, alluvions profondes des basses vallées, biefs épargnés par les crues. La diversité spécifique est de plus en plus faible lorsque la continuité temporelle (horizon superficiel des zones à forte pente) et la continuité spatiale (morcellement et isolement des biotopes) diminue. A la limite, le peuplement prend un caractère aléatoire.

\section{8. - Conclusions}

Cet te étude des Hydracariens des Pyrénées centrales et des Coteaux de Gascogne nous a permis de mettre en évidence un certain nombre d'associations. Celles-ci sont corrélées avec des paramètres hydrologiques et topographiques, - régime des eaux, altitude de la source et de la station, pente, surface du bassin versant, substrat, - qui constituent un complexe stationnel aux caractéristiques indépendantes des variations saisonnières.

Ces paramètres rendent compte de facteurs écologiques comme le nombre de degrés-jours, l'instabilité du substrat. Parallèlement, des types biologiques d'Hydracariens ont été déterminés, en fonction du cycle biologique, du taux de reproduction, de la nature du substrat.

Parmi les facteurs écologiques qui sont à l'origine de la sélection de certains types biologiques figure d'abord le régime des eaux. L'écoulement des eaux de pluie peut être immédiat ou différé (neige) d'un cours laps de temps : ce sont les régimes pluvial ou pluvio-nival, à hautes eaux d'hiver ou de début de printemps. Le cycle des Hydracariens (du développement de l'ceuf à la ponte des femelles) se déroule du printemps à la fin de l'été (la plupart des Aturus Kongsbergia, Hygrobates calliger, Atractides nodipalpis, Torrenticola similis,...). Le cycle est retardé en altitude.

Les régimes nival ou nival de transition sont caractérisés par une crue de printemps et début d'été, résultant de la fonte des neiges. Cette période correspond à une phase de repos de la faune. Le cycle des Hydracariens se déroule alors de la fin de l'été à la fin de l'hiver, - c'est-à-dire à basse température. Enfin, sur les cours d'eau à étiage d'été accentué (régime pluviothermique des rivières gasconnes), un certain nombre d'especes (appartenant surtout au genre Torrenticola) peuvent subsister en se développant au cours de l'été.

Le substrat joue ensuite un rôle décisif. Comme dans les sols terrestres, on peut distinguer les mousses, l'horizon superficiel des galets (litière), le milieu hyporhéique de surface (milieu édaphique) et de profondeur (milieu endogé). A chacun de ces biotopes correspond un type biologique d'Hydracariens.

Sur une rivière ayant atteint son profil d'équilibre, les mousses se développent sur le cours supérieur, fixées sur des substrats stables (roche-mère, 
blocs de rochers) non remaniés par les crues. Puis apparaissent les galets, et l'épaisseur de leurs dépôts s'accroit dans la basse vallée. L'horizon superficiel des fonds de galets est instable, susceptible d'être remaniè à chaque crue. Par contre, en profondeur, la stabilité dans le temps du milieu hyporhéique est assurée.

Dans l'horizon superficiel des fonds de galets vivent des espèces opportunistes, - Sperchon, Lebertia, Hygrobates, Atractides du groupe gibberipalpis-nodipalpis. La pente joue un rôle fondamental dans leur répartition.

Les Hydracariens muscicoles vivent essentiellement dans les Hypnacées, mousses couchées sur leur substrat et dont les feuilles, en forme de cupule, assurent une protection contre le courant aux espèces de petite taille. A la continuité dans le temps du biotope correspond un nombre d'ceufs réduit et la présence simultanée de plusieurs espèces d'un même genre dans le biotope (Feltria, Aturus), - probablement en fonction de ressources diversifiées.

Enfin, le milieu hyporhéique est colonisé par des espèces qui l'habitent de façon préférentielle (Torrenticola,...) ou stricte. A la continuité dans le temps du biotope correspond également la réduction du nombre d'œufs par femelle. Les lignées muscicoles et hyporhéiques sont souvent proches sur le plan phylétique (genres Feltria, Aturus, Kongsbergia, Alractides du groupe loricatus,...).

Aux successions de substrats sur le profil en long d'une rivière correspondent par conséquent des types biologiques d'Hydracariens, de l'amont vers l'aval :

- association muscicole - association de galets associations de galets + association hyporhéique.

Les espèces constituant ces associations sont évidemment différentes suivant le régime des eaux :

- en régime nival, Feltria muscicoles et Atractides gibberipalpis-Sperchon denticulatus dans l'horizon superficiel des galets.

- en régime pluvio-nival ou pluvial, AturusKongsbergia muscicoles et Atractides nodipalpisSperchon clupeifer dans l'horizon superficiel des galets.

Les successions d'espèces amont-aval que révèle l'analyse factorielle des correspondances correspondent à un classement. Il est plus juste de parler de types biologiques et de spectres biologiques corres- pondant à des complexes stationnels.

Ainsi, une station de piémont sans crue hivernale accusée pourra héberger des espèces de régime pluvial en été et de régime nival en hiver. Une retenue détournant la crue de printemps permet le développement d'espèces de régimes pluvial et nival.

L'étude de la structure des communautés d'Hydracariens montre une diversité croissante de l'amont vers l'aval, plus élevée également dans les mousses que dans l'horizon superficiel des galets. L'indice de dominance et les diagrammes rangfréquence traduisent la dominance d'un nombre réduit d'espèces sur le cours supérieur (écosystèmes pionniers), tendant vers l'équifréquence d'un certain nombre d'espèces de taille voisine sur le cours inférieur (écosystèmes matures, ou climaciques).

Cependant, il existe un petit nombre de stations privilégiées, dans lesquelles le nombre d'espèces se situe très au-dessus de la moyenne, à indice de diversité élevé et indice de dominance faible. Ces stations sont caractérisées par une surface importante et l'absence de facteurs écologiques dominants (crues détournées ou diminuées, pente faible, forte épaisseur des alluvions. La continuité du biotope est assurée à la fois dans l'espace et dans le temps. Chaque station héberge trois à quatre associations d'Hydracariens, correspondants a autant de types biologiques, et habituellement séparées.

Lorsqu'on remonte une rivière vers sa source, la pente devient de plus en plus forte, et certains types biologiques sont ainsi éliminés. De plus, les biotopes sont de plus en plus morcelés : la continuité spatiale diminue de l'aval vers l'amont.

Le mode de peuplement des eaux courantes par les Hydracariens s'apparente à celui des mousses corticoles par les Thécamoebiens, et, plus généralement, à celui des îles, réelles ou virtuelles. Le nombre d'espèces d'un biotope est d'autant plus réduit que sa surface est plus faible et son isolement plus grand. A la limite, - dans les sources, par exemple, $\rightarrow$ le peuplement peut devenir aléatoire. Le fait que, dans les biotopes de haute altitude à surface exceptionnellement élevée (Mousquere sur le plateau d'Arsoué,...), la communauté se rapproche du type climacique appuie cette conception. 
Travaux cités

Angelier (C.). - 1950. - La faune Hydracarienne du Massif du Néouvieille. Bull. Mus. Hist. Nat. Paris, 22, $3: 232-237$

Angelier (E.). - 1953. - La notion de frontière biogéographique Ouelques exemples de répartition des Hydracariens dans les Pyrénées. C.R. Soc. Biogéogr., $262: 47-47$.

Angelier (E.). - 1957. - La répartition des Hydracariens dans les Pyrénées et la notion de frontière biogeographique. Abh. Nat. Ver. Bremen. 35 (1): 2430 .

Angelier (E.). Decamps (H.) \& Rey. - 1963. - Les Hydracariens du Céret : étude systématique et écologique. Built. Soc. Hist. Nat. Toulouse, $98: 459-500$.

Bader (C.). - 1968. - Verläufige Resultate einer jahreszeitlichen Untersuchungen an Bachhydracarinen. Rev. Suisse Zool., 75 : 498-505.

Bonnet (L.). - 1973. - Le peuplement thécamoebien des mousses corticoles. Protostilogica, $9: 319-338$.

Bouguenec (V.), Meurgues (S.) \& Sourzac (C.) - 1984. Introduction à l'étude d'une rivière pyrénéenne aménagée : La Neste d'Aure. Mém. D.E.A. Ecologie, Toulouse : 158 p.

Cuinat (R.). - 1971. - Principaux caractères observés sur cinquante rivières à truites françaises. Influence de la pente et du calcium. Annls. Hydrobiol, 2 : 187-207.

Dawson (F.H.). - 1973. - Notes on the production of stream bryophyes in the high Pyrenees (France). Annls Limnol., 9: 231-240

Decamps (H.) - 1967. - Ecologie des Trichoptères de la vallée d'Aure (Hautes-Pyrénées' Ann's. Limnol. 3. 399.577

Devaux (J.7. - Intérét de l'utilisation des diagrammes de Frontier pour délimiter les stades des successions phytoplanctoniques. C.R. Acad. Sci., Paris, sér. D, 282 : 1499-1501.

Frontier (S.). - 1977 a. - Réflexions pour une théorie des écosystèmes. Bull. Ecol., 8 : 445-464.

Frontier 1977 b. - Utilisation des diagrammes rang-fréquence dans l'analyse des écosystèmes. Bull. Rech. Océanogr., 1 (3): $35-48$.
Gazagnes (G.). - 1983. - Contribution à l'étude de la dérive des Invertébrés sur la haute Neste d'Aure : impact des aménagements hydróélectriques. Thèse spécialité Ecologic, Toulouse : $104 \mathrm{p}$.

Huet (M.). - 1946. - Note préliminaire sur les relations entre la pente et les populations piscicoles des eaux courantes. Règle des pentes. Biol. Jahrb. Dodonaea, 13: 232-234

Huet (M.7. - 1949. - Aperçu des relations entre la pente et les populations piscicoles des eaux courartes. Schweiz Hydrobiol., $11: 332-351$

Lambert (R.). - 1975. - Recherches hydrologiques dans le Sudest du Bassin garonnais. These Univ. Toulouse II : $750 \mathrm{p}$.

Lauga (J.) \& Thomas (A.). - 1978. - Etude écologique des Aihericidae et Rhagionidae torrenticoles du sud de la France par l'analyse factorielle des correspondances. Bull. Sac. Hist. Nat. Toulouse. $114: 274-287$.

Lavandier (P.) \& Dumas (J.). - 1970. - Etude écologique de la faune aquatique de la Cressonière d'Agos (Hies-Pyrénées: cycles de developpement. Répartition. Thèse de spécialite Hydrobiologie, Toulouse : $158 \mathrm{p}$.

Mac Arthur (R.) \& Wilson (E.O.). - 1963. - An equilibrium theory of insular zoogeography. Evolution, $17: 373-387$.

Mac Arthur (R.). - 1967. - The theory island biogeography. Prince un Univ. Press, New Jersey : 203 p.

Mandelbrot (B.). - 1953. - Contribution à la théorie mathématique des communications. Thèse Univ. Paris. Pubi. Inst. Stat. Univ. Paris, 2, 1/2:121 p.

Mattei (G.). - 1966. - Les Hydracariens du Massif du Néouvielle. D.E.S. Fac. Sci. Toulouse : $36 \mathrm{p}$.

Motas (C) - - 1928 - Contribution à la connaissance des Hydracariens français, particulièrement du sudest de la France. Rev. Lab. Hydrobiol. Pisc. Univ. Grenoble, 20 : 1.370.

Thienemann (A.) - 1939. - Grundzüge einer allgemeine Ökologie. Arch. Hydrohiol., $35: 267.285$

Travers (A). - 1971 - Diversité du microplancton du golfe de Marseille. Mar. Biol., 8 : 308-343.

Thomas (A.) - 1976. - Diptères torrenticoles peu connus : IV. Les Athericidae (ecologie et biologie) du sud de la France (Brachycera, Orthorhapha). Annls. Limnol., 12 : 175-211.

Vincent-Genod (R.). - 1963. - Contribution à l'étude de la faune du ruisseau d'Espiaube (Hydracariens et Coléoptères). D.E.S. Fac. Sci. Toulouse : 37 p. 